THESIS

\title{
MODALITY SWITCHES OCCUR EARLY AND EXTEND LATE IN CONCEPTUAL PROCESSING: EVIDENCE FROM ERPS
}

\author{
Submitted by \\ Pablo César de Juan Bernabéu
}

In partial fulfilment of the requirements for the degree of

Research Master (Mphil) in Language and Communication

Tilburg University

Radboud University

March 2017

Supervisors:

Assoc. Prof. Roel Willems (Centre for Language Studies, Radboud University)

Prof. Max Louwerse (Centre for Cognition and Communication, Tilburg U.) 


\section{Acknowledgments}

I would like to first thank both of my supervisors for their trust and support. Being able to conduct this experiment has been very valuable and enjoyable to me. Thanks also to the Neurobiology of Language department of the Max Planck Institute for Psycholinguistics for offering their resources so openly. I was amazed by the human support and the technical facilities. Thanks also to the Experimental Psychology Society for a conference presentation grant.

Other experts who have helped me with their advice and technical know-how include Charlotte Poulisse, Diane Pecher, Franziska Hartung, Gwilym Lockwood, Johan Weustink, Kara Federmeier, Martijn Goudbeek, Monique Flecken, Ronald Fischer, Sean Roberts, the support staff of Neurobehavioral Systems and Brain Products, and several contributors on the internet.

Further off the Psycholinguistics field, I am also grateful to Ad Backus and other organizers of the master's, to Omid Feyli, and to over a hundred students from Nijmegen and Tilburg who kindly participated in the research at different stages. 


\section{Modality switches occur early and extend late in conceptual processing: Evidence from ERPs}

The engagement of sensory brain regions during word recognition is widely documented, yet its precise relevance is less clear. It would constitute perceptual simulation only if it has a functional role in conceptual processing. We investigated this in an Event-Related Potential (ERP) experiment implementing the conceptual modality switch paradigm. In each trial, participants verified the relation between a property word and a concept word. Orthogonally, we manipulated the conceptual modality of successive trials, and tested whether switching modalities incurred any processing costs at different stages of word recognition. Unlike previous studies, we time-locked ERPs to the first word of target trials, in order to measure the modality transitions from the beginning, and also to reduce confounds within the target trial. Further, we included different types of switch-one from auditory to visual modality, and one from haptic to visual-, which were compared to the non-switch-visual to visual. Also, one group of participants was asked to respond quickly $(n=21)$, and another group to respond self-paced $(n=21)$, whilst a few others received no constraints $(n=5)$. We found ERP effects in four typical time windows from 160 to $750 \mathrm{~ms}$ post word onset. The overall effect is characterized by a negativity for modality-switching relative to not switching, and it increases over time. Further, the effect arises with both types of switch, and influences both participant groups within anterior and posterior brain regions. The emergence of this effect in the first time window particularly suggests that sensory regions may have a functional role in conceptual processing. The increased effect later on converges with previous studies in supporting the compatibility of distributional and embodied processing. On a less conclusive note, more research may be necessary to ascertain the nature of the effect at late stages. 


\section{Introduction}

How do we understand words? Research suggests that there may be two mental systems at play. One of these systems works by relating a given word to related concepts. For instance, suppose you are checking your schedule for next week, and you read the word 'Trip.' This would instantly prompt in your brain connections to relevant concepts such as travel, luggage, train, ticket, etcetera (subject to context). The activation of such a network of concepts would commonly build up the meaning of the word for you. The second system is instead based on the activation of senses, actions, and emotions that are relevant to a given word. In this system, the relations are not merely distributional but are specifically set within the same modalities of the original word. Thus, your reading of 'trip' could activate neurons that code for the sensation of being surrounded, the action of walking, the feeling of excitement, etcetera. The re-enactment of such embodied experiences would yield the meaning of the word. The primary root of both systems is of course language. Likewise, context and one's own experience is important to both systems. What distinguishes the two systems is their connections: While the 'distributional' system works on rather abstract, heuristic connections based on linguistic symbols, the 'embodied' system implements connections that are more directly based on one's own physical self.

The nature of conceptual processing has occupied research in psycholinguistics and cognitive neuroscience for over four decades. In this article, we wish to contribute a literature review and an experiment. In the review, we will go over the evidence for distributional processing and for embodied processing. We identify the main challenges in testing each type of process, then point out some methodological approaches that may prove helpful. Specifically, the evidence on distributional processing will be increasingly compelling if it continues to include a priori predictions in detail. Likewise, the evidence on embodied processing will benefit from measuring the time course of sensory-motor engagement with increasing precision. Yet, each consideration indeed applies to both systems more generally. Last, we note the increasing evidence for the compatibility of distributional and embodied processes. After the review, we present an electroencephalographic experiment in which we tested the embodied system with a precise timing. As results, we found an effect of perceptual simulation starting as soon as a word is recognized, and increasing throughout thereafter. The results suggest that 
sensory brain regions contribute to the core processing of concepts. In closing, we discuss some challenges and possible directions in this topic area.

\section{Literature review}

The study of thinking has long contemplated the possibility that higher cognition might be fundamentally based on one's own physical self. For instance, the way we understand an auditory property such as 'loud' could be enabled by our sense of hearing. In spite of the longevity of this consideration, there was an important period in contemporary science where that relation was disregarded or denied, in favour of a higher cognition that would be based on rather abstract, arbitrary relations (Barsalou, 1999).

\section{Early accounts of amodal conceptual processing}

During the Cognitive Revolution, spanning the second half of the $20^{\text {th }}$ Century, higher cognitive processes such as meaning comprehension were regarded as fundamentally disconnected from perception. This was greatly due to the successful development of computers, which had demonstrated that a system without percepts could satisfactorily operate. Thus, by the same token, the human conceptual system might possibly process concepts on the basis of amodal symbols, as long as they were robustly interconnected. ${ }^{1}$ The brain does certainly possess a broad connectivity. On this account, people would understand words through a process of association within the mental lexicon, such that a word would have meaning insofar as all other words had meaning (Rumelhart, McClelland, \& the PDP Research Group, 1986; for a review, see Barsalou, 1999).

The main influences in the research of the time were computing, logic, predicate calculus, and connectionism. They all implemented devices such as binary values and logical operators. Some of those constructs have proven useful in the study of conceptual processing, and still continue to evolve. For instance, Barsalou (1999) highlighted two of those contributions. The first of those were some requirements posited for a viable conceptual system, including an 'ability to represent types and tokens, to produce categorical inferences, to combine symbols productively.' The second contribution were

\footnotetext{
${ }^{1}$ Standard terminology is used in this article (see Barsalou, 1999; Mahon \& Hickok, 2016). First, the term 'modality' refers to conceptual modality, rather than presentation modality. The terms 'amodal' and 'distributional' are used almost indistinctly, and so are 'modal' and 'embodied' at the other end of the spectrum. Last, the term 'symbol' denotes the potential neural substrates for conceptual processing, that is, the representational format (see also Mahon \& Hickok, 2016).
} 
research techniques based on cognitive models of the era, including 'feature lists, frames, schemata, semantic nets' (Barsalou, 1999, pp. 578, 579; see original constructs in Fodor \& Pylyshyn, 1988; for a renewed use, see Santos, Chaigneau, Simmons, \& Barsalou, 2011). Those contributions were recognized, and somehow implemented, by a set of cognitive scientists who nonetheless would propose a different approach to higher cognition. For instance, Barsalou (1999) and Pulvermüller (1999) demonstrated that mental symbols of a perceptual nature could enter logical relations, in a similar guise as amodal symbols. Most notably, these novel proposals reclaimed the attention to experiential systems of perception, action and emotion, which had been lacking.

\section{Early accounts of modal conceptual processing}

Barsalou and Pulvermüller contended that amodal, or connectionist, accounts had sidestepped modal brain systems before actually having a robust enough case for amodal systems. Barsalou specifically highlighted the fact that no research had demonstrated how a perceptual state - for instance, the perception of a landscape or a scent-could be transformed into an amodal symbol in the brain (a process dubbed 'transduction'). Yet, even if such an ontogeny were ever provided, there would be problems in accounting for processes on-line because of the symbol grounding problem. A conceptual system based on amodal, possibly linguistic, associations would ultimately suffer from lack of reference to the real world. This problem was famously exemplified in a thought experiment known as the Chinese Room (Searle, 1980). An English speaker who has no idea of Chinese seemingly manages to answer questions in Chinese. The feat is achieved as follows. The experiment participant sits in a locked room which is connected to the outside through two grooves on opposite sides of the room. In each trial, the experimenter slips in a batch of three files to the participant, who must use them to produce meaningful answers. Upon completion, he will slip out the questions and the answers through the opposite groove, where naïve speakers of Chinese will evaluate them. The input in each trial is as follows: a set of questions in Chinese, an answer sheet, and a simple grammar of Chinese written in English. This grammar only contains ad-hoc rules for interpreting the Kanji. The participant manages to do so, as confirmed by the Chinese speakers who receive his output. However, he still has not understood a word of the input or of his own output. This script could serve as a metaphor for amodal conceptual processing, suggesting that distributional associations can only virtually work. In human rather than artificial 
intelligence, they would prove insufficient because the activations they produce would not be grounded in the real world. The most basic binomials in language would lack a half: the sign would have no referent, the parole would have no langue (Saussure, 1916/1959).

The new, modal symbol theories actually went further than reclaiming attention to the neural systems for perception, action, and emotion. They argued that those systems were the basis of higher cognitive processes. In this way, the process of 'transduction' from perceptual states to symbols would no longer be necessary. The symbols would simply take the same form as the percepts because they would be encoded on the same substrates in the brain. One other advantage for this account was that more was known about the structure and function of modal regions (e.g., the homunculus, found by Penfield \& Boldfrey, 1937) than about their amodal counterparts. Furthermore, some of these abstract areas, known as convergence zones, feed on different modal areas to create more abstract content. Thus, even the output of those in principle amodal areas would be bound to bodily systems (Barsalou, 1999). Owing to the cortical substrate of the symbols, the new account became known as embodied cognition, or 'embodiment.'

Pulvermüller, Barsalou, and the respondents to their target articles put forth the directions that have occupied research up to this date. Besides discussing the relevance of lowerlevel systems for processes such as word comprehension (cf. Hauk, Johnsrude, \& Pulvermüller, 2004; see also Simmons et al., 2007), they entertained topics such as the individual differences that should follow from such a hypothesis.

Well supported yet provocative, the embodiment proposal was met with considerable criticism. For instance, Landauer (1999) claimed that it was critically challenged by the research in computational linguistics. He referred specifically to Latent Semantic Analysis (LSA), a technique that can compute the relation among any linguistic units (e.g., words) on the basis of higher order corpus relations (that is, any co-occurrences, however removed). To use the tool, one may input any series of words, and will receive as output a matrix containing the relationship of every inputted unit with each other. ${ }^{2}$ For each relation there is a score that may range from 0 - for totally unrelated units - to 1 for the exact same units. Landauer's title was clear enough: 'Latent Semantic Analysis

\footnotetext{
${ }^{2}$ Freely available online at http://lsa.colorado.edu.
} 
(LSA), a disembodied learning machine, acquires human word meaning vicariously from language alone' (similarly, see Lund \& Burgess, 1996). In turn, Barsalou (1999) countered that such computational re-analyses could explain any finding post-hoc, but they failed to posit enough theory-driven predictions (see also Anderson, 1978). We will see below that computational approaches have increased their precision.

The other major source of criticism on embodied cognition concerned the allegedly functional role of sensory, motor, and emotional systems for conceptual processing. Critics doubted that the engagement of such systems was indeed causative of conceptual processing. This engagement could instead be a consequence of conceptual processing. For their part, the proponents of embodied cognition did not deny such a potential, ulterior engagement, but made the point clear that embodied cognition was made up of the prior, causative engagement (Barsalou, 1999; Pulvermüller, 1999). The implications were that embodiment effects could only be noted as such so long as they were traceable down to a point in the causality or the time scale at which they clearly had not been not effected by the core of conceptual processing, but rather had actively contributed to that process (Adams \& Campbell, 1999). Few hints were offered back then on how to control this, and time has indeed shown it to be a hard nut to crack. Yet, the condition was fair, as amodal symbol accounts were likewise expected to be falsifiable (Barsalou, 1999).

\section{Current state of affairs}

Although abundant knowledge has been gained since the early 2000s, the main lacunae on distributional and embodied processes remain. On the one hand, the formation of amodal symbols has not yet been demonstrated, nor has the referential grounding of such symbols (Barsalou, 2016). On the other hand, the causality of sensory or motor engagement, with regard to core meaning comprehension, has not been ascertained either (Mahon \& Hickok, 2016). Traditionally, more attention has been focused on the caveats of embodied processing, and there might be reason for this imbalance.

At the debut of the research on embodiment, Barsalou (1999) criticized a widespread bias in the field by which amodal processing would be considered as the default system, even in advance of enough evidence. By contrast, the same field would demand more conclusive evidence for embodiment. This bias merits some analysis because it may persist to date. Such a line of thought may respond to the relative accessibility to modal 
symbols compared to amodal ones. Modal symbols present themselves as easy to pin down and test, unlike amodal ones. If cognition was embodied, we should find action concepts being processed in premotor and primary motor cortices, and perception concepts being processed in primary perceptual and somatosensory cortices. By contrast, the predictions we can make for an amodal account in terms of brain sites are more limited. For instance, action and perception concepts would be predicted over supramodal regions, allowing less precision than the relevant modal regions would allow (see Binder \& Desai, 2011). Furthermore, if we consider the other sources of evidence, behavioural and computational, we find more justification. The power of the distributional system to challenge the results of embodiment is well known (e.g., Louwerse \& Connell, 2011). Yet, there are arguably fewer if any studies re-analyzing evidence for distributional processing in terms of embodied processing. Last and most general, we count on a much more extensive body of contemporary research in neuroscience within a computational framework than within anything like an embodiment framework. For all of these reasons, embodied processing may have come across, up to this date, as a more inviting target for testing than distributional processing.

Amidst growing attention to the evidence on embodiment, and its potential power, some researchers have called for a measured, critical standpoint about it (Willems \& Francken, 2012; Louwerse, 2011). This caution is mainly due to inconclusive evidence on embodiment, and to evidence for a compatibility of distributional and embodied processing. We tackle each in turn.

Inconclusive evidence

Let us start with patient studies. Among those, we find on the one hand results that support the relation of sensory-motor systems with conceptual processing, whilst on the other hand we find the opposite. For example, Grossman et al. (2008) found a correlation between motor cortex atrophy and difficulty to understand action words. Similarly, a study with stroke patients found that motoric ability predicted motor-related conceptual processing (Desai, Herter, Riccardi, Rorden, \& Fridriksson, 2015; see also Kiefer, Sim, Herrnberger, Grothe, \& Hoenig, 2008). In contrast to the latter, Negri et al. (2007) found no significant role of unilateral stroke on conceptual motor tasks. The authors specified that a significant motor-conceptual correlation was found at the group level but not at the single-case level (see also Garcea, Dombovy, \& Mahon, 2013; Vannuscorps \& 
Caramazza, 2016).

Non-clinical experiments are the second broad area presenting mixed results. Over a decade past the finding that physical motion and motion words engage motor cortex similarly (Hauk, Johnsrude, \& Pulvermüller, 2004; see also Simmons et al., 2007), the current state of affairs is directing us to investigate the precise function of any sensorymotor correlates. Indeed, for all their value, fMRI studies such as those are liable to skepticism due to the low temporal resolution of the measurements, and hence difficult causal inferencing. In the processing of single words, every millisecond counts. That said, we must acknowledge at this point that we do not know either when exactly the core processing of a concept ends and the less core processing starts. For instance, in the area of single word comprehension, we might posit that by about $550 \mathrm{~ms}$ post-word onset, the core meaning of a content word has been processed but we do not know with certainty. We can only work with the reasonable assumption that the earlier the sensory-motor engagement, the more likely it contributes to the core of conceptual processing-or vice versa, the less likely it reflects post-conceptual mental imagery (see further details below). As Hauk (2016) reviews, this assumption is one of the backbones of current research (see also Hauk, Coutout, Holden, \& Chen, 2012; Willems, Toni, Hagoort, \& Casasanto, 2010; Hauk, Shtyrov, \& Pulvermüller, 2008). We must, however, be explicit that the 'core' of conceptual or semantic processing is a relative matter. An absolute core might not exist, as conceptual processing (and language comprehension, more generally) is an extended, incremental process which starts small but grows in a very complex manner-even in the lab (Lebois, Wilson-Mendenhall, \& Barsalou, 2014). Yet, the 'core' of the process can arguably be a useful guide for weighing the role of experimental effects, if it is studied with enough control and in a relative manner.

Time course of single word processing. The association of earlier semantic processes to a greater relevance for comprehension is supported by some evidence. In a nutshell, broader processes start only after more immediate ones have started. The most immediate process is the recognition of a string of letters as a word, which seems to start within 90 ms post word onset in early auditory cortex and the Visual Word Form Area (Willems, Frank, Nijhoff, Hagoort, \& van den Bosch, 2016). Broader lexical and semantic processes ensue then, namely, identification of the word as a known word within around $160 \mathrm{~ms}$, and access to its meaning within around $200 \mathrm{~ms}$ (Hauk, 2016). These processes may overlap, as the sensitivity of the N400 to both suggests (Kutas \& Federmeier, 2011). They 
also likely extend further in the processing timeline, albeit with a lesser role. The lexical and semantic stages are directly relevant to our current topic, as shown in studies with different processing tasks. Tasks promoting semantic processing seem to engage embodied systems more than lexical tasks do (Sato, Mengarelli, Riggio, Gallese, \& Buccino, 2008).

Once the lexical and semantic stages have emerged, post-lexical, post-semantic processes will emerge. These are mental imagery and episodic memory processes - both with an approximate emergence around $270 \mathrm{~ms}$ after word onset. The gradual progression from the identification of a word up to accessing its broadest meaning is an important anchoring point in the current research on the alleged embodiment of meaning comprehension, even if we would rather count on more definitive threshold points (Hauk, 2016).

Word processing data are mainly based on written word processing, but spoken words are processed quite similarly, if slightly faster (Leonard, Baud, Sjerps, \& Chang, 2016; Pulvermüller, Shtyrov, \& Ilmoniemi, 2005; Shtyrov, Hauk, \& Pulvermüller, 2004). The bigger take-home messages would be: (1) the processing of meaning might only start at around $160 \mathrm{~ms}$ after word onset, and (2) processes outside of meaning comprehension might only start at around $270 \mathrm{~ms}$ after words onset. These working references must be taken with some caution because particular semantic effects have been found at different stages (e.g., the conceptual modality switch, as in Hald, Marshall, Janssen, \& Garnham, 2011; Collins, Pecher, Zeelenberg, \& Coulson, 2011). Indeed, in an influential critique of blooming findings on embodiment, Mahon and Caramazza (2008) argued that even early effects might possibly be explained in terms of non-embodied processing. They contended that working memory processes that were ancillary rather than semantic could be quickly engaged with the function of 'colouring' a concept, not building it up. Yet further, neither do we have absolute certainty on the later section of the time course. As Hauk (2016) reviews, the different stages likely overlap at certain points, with different degrees of relevance. For instance, lexical processing may continue even once semantic processing has started, but would naturally become less relevant. Indeed, the relation among these processes is likely more of a continuum than a set of clear-cut modules. In sum, it seems that much of the cause why the current topic does not quite get resolved is the fundamental lacunae we have on the time course of meaning comprehension in language. 
Caution notwithstanding, we may have to work with what we have. For instance, since modal systems appear to take place later in word processing, as compared to the earlier peak of amodal systems, a threshold around $270 \mathrm{~ms}$ post word onset could prove useful in testing the contribution of modal systems. Provided a temporally-precise design and measure, with reasonable margins around the time landmark, there would be two possible results: modal systems are engaged before $270 \mathrm{~ms}$ post-word onset, or afterwards. The former result would suggest the contribution of those systems to meaning comprehension (provided a reasonable margin from the exact threshold, of course). The latter result, by contrast, would be less definitive. Simply, it would lend itself to the relative considerations that reign in the current field: the earlier, the more likely modal systems have contributed to meaning comprehension. Regardless of the result, these measures would have to be taken via EEG or MEG, and the specific engagement of modal systems would be determined by a sensory, motor, or emotional effect on a particular time window. Time windows should be chosen in accord with the aforementioned thresholds, approximately (for instance, see Popp, Trumpp, \& Kiefer, 2016; Hald et al., 2011). Also very important, the engagement of whatsoever systems would have to be elicited and measured with a causal articulation. This point was famously highlighted by Mahon and Caramazza (2008), as they criticized designs that measured activity rather in raw. For instance, Hauk and Pulvermüller (2004) found that premotor cortex for the leg, the arm, and the face selectively responded to the corresponding action words (e.g., 'walk, 'wave,' 'smile') within $200 \mathrm{~ms}$ from the onset of those words (see also Pulvermüller, Shtyrov, \& Ilmoniemi, 2005). Although the quickness of that engagement might suggest a functional role for motor regions, Mahon and Caramazza countered: 'How else (but somatotopically) could the motor system be activated?' (p. 62). The main focus of their critical review was indeed the causality of the effects found in the embodiment studies. More conclusive results seemed to be necessary, possibly by means of more elaborate measures.

When considering fMRI evidence, we must acknowledge the possibility that sensorymotor correlates had rather been 'peripheral and epiphenomenal to the representation itself' (Monaghan \& Nazir, 2009, p. 337). Crucially, if we consider this post-core activation, we will likely agree that that alone would be useful for behaviour, as it would quickly enable physical memories about a concept, and furthermore activate the relevant areas for any sensory-motoric responses that might obtain. This kind of non-core but near- 
immediate engagement of sensory-motor systems is widely acknowledged (Mahon, 2015). However, we still have to elucidate the relevance of those systems for the core of conceptual processing, for instance, for the understanding of meaning in language. To this aim, Chatterjee (2010) proposed looking at the data more closely, at the neuron level, because the allegedly 'embodied' engagement often occupies multiple regions. For instance, Tettamanti et al. (2005) reported that action sentences engaged premotor, intraparietal sulcus, and inferior parietal cortices. Yet, even though the three regions appeared to take up a common functional goal at the presentation of the stimulus, it might be best to double-check whether the neurons in those regions also act in unison. The degree of functional isomorphism therein would determine the robustness of the findings.

In behavioural experiments, the major challenge is again determining the relevance of any sensory or motor engagement to core conceptual processing. Measures of response time, accuracy and response choice are taken during or after the comprehension of the stimulus in each trial. This is necessarily so because a participant cannot legitimately respond before understanding the basic meaning of the stimulus. In the literature, this seems to raise less concern than it should. It may possibly be assumed as a natural characteristic of behavioural measures. Granted, these behavioural measures in principle guarantee a robust link between stimulus and reaction. However, this is less true when we are dealing with words. The core meaning of a word is processed within some 600 milliseconds (Hauk, 2016). For an illustration, consider an experiment featuring stimulus words which are typically related to a certain location, and hence also direction-e.g., 'star' (up), 'mole' (down). These relations are covert and orthogonal to the task, which in fact is simply to signal the font colour of the words. The way of signalling this upward or downward arm movements, as requested per trial. This is an interesting design, analogous to others such as the conceptual modality switch. In this experiment (Dudschig, de la Vega, \& Kaup, 2014), an interference effect was found whereby incongruent trials (e.g., having to move up for a downward word such as 'mole') incurred longer reaction times than congruent trials (for other experiments with behavioural measures, see Pecher, Zeelenberg, \& Barsalou, 2003; Solomon \& Barsalou, 2004; Richter \& Zwaan, 2010; Edmiston \& Lupyan, 2017). The sensory enactment effect rightfully seems to follow from the experimental manipulation. Yet, the time course of the effect does not allow strong enough inferences because the measure was taken after, rather than during, comprehension. The sensory enactment might therefore have been engaged only after 
comprehension of the stimuli. Indeed, another experiment, implementing a similar manipulation, found that embodiment effects tended to appear later in the trial (Louwerse \& Hutchinson, 2012; see also Louwerse, Hutchinson, Tillman, \& Recchia, 2015).

Behavioural and fMRI measures have an inherent time lag that allows criticism on the precise relevance of the effect to conceptual processing. The few hundreds of milliseconds that are unaccounted for are enough for later semantic processes to arise. Those later processes - imagery and episodic memory - may start from around $270 \mathrm{~ms}$ (Hauk, 2016). Once they have started, our certainty on the role of sensory-motor effects decreases.

There is a technique that is especially fit for testing the causality of cognitive systems. This is the modulation of neural resources. On this topic, this has been achieved via transcranial magnetic stimulation (TMS) specifically, in studies which focused on the embodied system. For instance, Papeo et al. (2009) applied TMS on motor brain regions, immediately presenting participants with verbs related to hands and feet movement. The authors then measured motor-evoked potentials (MEPs) in the hands and feet, as a proxy for activity in the corresponding brain regions (similarly, see Buccino et al., 2005). Problematically, however, those measures present the same caveats above exposed for any off-line, or on-line but lagged, measurements. The results do not conclusively point to a functional role of embodiment systems in conceptual processing because of the notably lagged measurement. The MEPs might as well follow from an understanding of the stimuli rather than reflect an on-line engagement of primary motor or premotor cortex. To solve that, Willems et al. (2011) measured used TMS as well, but to modulate rather than measure neural activity. They then measured its influence on language comprehension. Specifically, the authors stimulated the hand area of premotor cortex. They then measured how participants comprehended actions that are typically involving the dominant hand, such as 'throw' or 'write.' In addition, they measured comprehension of non-manual actions, such as 'earn' or 'wander.' Furthermore, they crossed those factors with hand dominance, stimulating both the right and the left hemispheres of the righthanded participants. As predicted, the application of theta-burst TMS boosted the participants' responses to manual actions but to non-manual actions, and more so when the stimulation was on the hemisphere corresponding to the dominant hand. Such an experiment aimed to advance the current topic towards increasing detail (see also Willems $\&$ Francken, 2012). Yet, the measure taken in that experiment was an off-line one-a 
conscious response from the participant several hundreds of milliseconds after reading the stimulus words. More recently, Vukovic et al. (2017) watched the time course further than ever as they knocked out primary motor cortex within $200 \mathrm{~ms}$ from word onset. If we recall the time course of word processing, that seems a good moment to act upon. Vukovic et al. found an impairment in the processing of action-related words, along with an improvement in the processing of abstract words. The last study suggests that the contribution of embodied systems (in this case, motor ones) can emerge very early on. It is not alone at that. A minority of studies have already measured effects right at, or very close to, the experimental manipulation - that is, closer than $300 \mathrm{~ms}$. Their results underscore the need for speed. For instance, Amsel, Urbach, and Kutas (2014) measured Event-Related brain Potentials (ERPs) in a semantic decision task. The authors created a particular interference between the conceptual content of the stimulus words and their visual contrast on the screen. They found a precise interference manifesting already 200 ms after word onset. In light of these findings, and the time course of word processing, we would conclude that experimental effects may need to be measured from the onset of the manipulation. For this, ERPs seem the most apt method, whenever the experimental design does not require high temporal resolution.

A review of this literature reveals that the causality is the critical question. On paper, it has been like that since Barsalou (1999), but research is a matter of time. The time course of single word comprehension may provide a helpful gateway in this quest. Accordingly, we may have to reconsider the methods. Can they all answer the critical questions we face currently? Considering the importance of the time course, neither fMRI nor off-line behavioural measures seem ideal. In contrast, on-line measurements with high temporal resolution-i.e., Event-Related Potentials (ERPs), Event-Related Fields, and eyetracking - can cater for the speed of word comprehension. Among these techniques, eye tracking has arguably been less used because the measures do not offer the well-known signatures - 'components' - that are collected with encephalographic measures. At present, however, eye tracking may have much to offer as we strive to present more natural language in the experiments (e.g., van den Hoven, Hartung, Burke, \& Willems, 2016).

Beyond that, there exist techniques of more elaboration which might be even better. The first is a combination of neuro-modulation, for instance via transcranial magnetic stimulation (TMS), and the measurement of ERPs. This allows an active modulation of 
the resources of particular brain regions, and would thus be ideal for testing the contribution of modal or amodal systems. For instance, for a test on the amodal system, activity within the classic language areas in the left hemisphere could be modulated (for ERP evidence, see Louwerse \& Hutchinson, 2012). The second technique is a combination of fMRI and ERPs. This is a very recent development, and to our knowledge has not been implemented on this topic. It would be potentially very valuable, as it unites high spatial and temporal resolution.

The elusive causality caveat may even affect neurophysiological measurements with high temporal resolution, such as Event-Related brain Potentials (ERPs), if they do not measure manipulations from their precise onset. This may include the extant ERP experiments on the conceptual modality switch (Hald et al., 2011; Collins et al., 2011; see also Hald, Hocking, Vernon, Marshall, \& Garnham, 2013). In those experiments, trials presented first a concept word and then a property word. ERPs were time-locked to the property word, which may have left uncontrolled a switch produced already at the concept. Indeed, the property word was already supposed to be in the particular modality of the trial. That pitfall could have had two consequences: loss of power, and loss of certainty on the time course of the effect. Thus, in the current state of affairs, it might be fitting to create a design where ERPs are time-locked to the very first word in each trial. To be clear, the purpose of this relocation would not be to annul the possibility of postcore sensory processes, but to measure the modality switch doubtlessly from its start.

Compatibility of distributional and embodied processing

Earlier accounts on distributional processing (e.g., Rumelhart, McClelland, \& the PDP Research Group, 1986) and those on embodied processing (e.g., Barsalou, 1999) would set themselves altogether apart from each other. With such a precedent, perhaps the most notable trait of the state of affairs at present is the widespread acknowledgment that conceptual processing may operate on both kinds of systems (e.g., Mahon, 2015). For instance, Santos et al. (2011) found evidence for distributional processing preceding embodied processing. Specifically, in a property generation task (interesting also because it studies language production), they found that 'properties bearing a linguistic relation to the word for a concept were produced earlier than properties not bearing a linguistic relation' (p. 83; see also Simmons, Hamann, Harenski, Hu, \& Barsalou, 2008; Barsalou, Santos, Simmons, \& Wilson, 2008; Louwerse \& Jeuniaux, 2010). Converging evidence 
was found by Louwerse and Connell (2011) as they re-analyzed an experiment which had implemented the conceptual modality switch paradigm (Lynott \& Connell, 2009). Instead of considering the traditional conditions, switch versus non-switch, the authors distinguished three conditions: (1) a switch across linguistically related modalities (i.e., haptic to/from visual, and gustatory to/from olfactory), (2) any other switch (e.g., gustatory to/from visual, haptic to/from auditory), (3) a non-switch (e.g., visual to visual, haptic to haptic). The three divisions were borne out of corpus research in fact (specifically, Principal Components Analysis). ${ }^{3}$ They hypothesized that if conceptual processing entailed both language- and simulation- based systems, then the distributional system (also called 'linguistic' system, due to the relevance of linguistic frequencies) would miss the easier shifts. Conversely, the 'embodied system' would pick them all up. Yet, the greater precision of the latter system would come at the cost of longer processing time. Indeed, earlier evidence had shown that embodied processes arose later than distributional processes (Simmons et al., 2008). To test their hypothesis, Louwerse and Connell divided the responses into three equal subsets: one for slower RTs, one for medium ones, and one for faster RTs. Finally, they ran a regression on each subset, with both types of switch as predictors, and found that faster RTs were best predicted by the easier shifts, slower RTs were best predicted by the harder shifts, and medium RTs were predicted equally well. In another study, more support for the compatibility was found through a meta-analysis of effect sizes, as embodied effects appear to decrease in faster behavioural conditions (Louwerse, Hutchinson, Tillman, \& Recchia, 2015). The relation of quicker and slower processing to different processing modes makes sense. Quicker processing simply seems to be less detailed. Interestingly, language production presents a related (not identical) balance by which faster speech contains less lexical information (Cohen Priva, 2017). The Louwerse and Connell study, as well as the Louwerse et al. study, suggest that computational approaches can posit precise hypotheses a priori. Indeed, they are necessary, considering that the main criticism on them has usually been the explanation post hoc (Barsalou, 1999).

Some other data that we think supports the compatibility of modal and amodal systems includes: Varying sensorimotor engagement in language comprehension (Samur, Lai, Hagoort, \& Willems, 2015), varying abstraction in conceptual processing (Spunt,

\footnotetext{
${ }^{3}$ See converging evidence in Lynott and Connell (2013), and in Bernabeu, Louwerse, and Willems (in prep.).
} 
Kemmerer \& Adolphs, 2016), with more abstraction occurring later (Amsel, 2011), and interplay of emotional and symbolic components in communication (Mehu, 2015).

The compatibility is further supported by data on the structure and function of different brain regions. Binder and Desai (2011) reviewed neuroimaging of semantic memory, distinctly identifying modality-specific and non-specific cortex. The two kinds of cortex seem to interact systematically, resulting in a model as follows. Modality-specific cortex occupies the regions for perception, action, and emotion, whilst non-specific cortex is located at the junction of different modal regions, in 'convergence zones' (it is ironic that convergence zones feed on modal cortex). Convergence zones enable supramodal and multimodal conceptual functions such as social cognition and object recognition. To underscore the neat co-habitation of modal and amodal systems, it seems that modal regions enable varying degrees of concreteness, and amodal regions enable varying abstraction. This indeed points to a continuum from abstraction to perception. More than that, there seems to be a large continuum from amodal to modal systems. In the middle of that continuum, the two kinds of processes could share the floor, or perhaps compete. For instance, in the Louwerse and Connell study mentioned above, this mixed floor is likely patent in the subset of medium RTs). In accord with such a continuum, Simmons et al. (2008) reject a modular division of modal and amodal systems, considering the broad connectivity in the brain. Indeed, each system (i.e., set of regions) may well have some tasks of their own. Particularly, modal regions, after enabling perceptual, motor, or emotional simulation during the core of conceptual processing, may subsequently facilitate actual responses of perception or action by simply retaining or possibly increasing their activation.

The last potential support comes from a recent fMRI study in which hours of narrative text were mapped on the brain (Huth, de Heer, Griffiths, Theunissen, \& Gallant, 2016). Words were found to cluster around categories: social words adhered to each other, tactile words too, and so on up to 12 categories. The results were as fascinating as they were daunting because the categories presented extremely broad distributions over the brain (similarly, see Anderson et al., 2016). For instance, tactile concepts show up in frontal, temporal, and parietal cortices - specifically, in superior and inferior prefrontal, lateral and ventral temporal, and lateral and medial parietal cortices. Likewise, visual concepts appear in primary visual cortex as well as in areas that are not visually specific. If we bring these findings to bear on our question, we could tentatively regard them as support 
for a compatibility of distributional and embodied processing, as we see that concepts take over both modal and amodal regions. This cannot be taken as uniquely amodal processing because we then have a large body of evidence for modal processing whichalthough inconclusive - has not been refuted.

Overall, the compatibility of a distributional and an embodied system is supported by neuroimaging, behavioural, and computational data (see also Andrews, Frank, \& Vigliocco, 2014; Dove, 2014). In more general terms, this hypothesis is compelling for two reasons. First, if each single account is weak to some extent, as we saw above, the two might offer more power in combination. Second, and more compellingly, reliable evidence for the compatibility would logically cancel the challenges of each single account, provided that no alternative accounts exist. Indeed there seem to be no alternative accounts at present. So quite interestingly, the compatibility findings represent a situation where the amodal and the modal systems serve as witnesses to each other. This evidence would thus be the strongest case for each system, on logical grounds. However, we must be cautious because much of the evidence for the compatibility is directly based on earlier experiments which did not control the embodied system far enough. The major caveat is the time course, as discussed above. This would be the case of Louwerse and Connell (2011). As described above, the authors implemented computational linguistics methods to re-analyze a previous experiment that had implemented the conceptual modality switch. Said experiment, by Lynott and Connell (2009), had implemented the classic design in the paradigm. Each trial presented a sentence: $A$ [concept] can be [property]. Orthogonally, trials were arranged to yield modality shifts and non-shifts. A property verification task then served to measure RTs. The problem here is that the measure was off-line, which affects the reanalysis in Louwerse and Connell by the same token. ${ }^{4}$ Within the off-line nature of the measure, the later the RTs the greater the risk of post-semantic processes causing the effect. Since they found that the embodied system responded later, that system would be under the main risk.

\section{Experiment}

In demonstrating the relevance of embodied cognition, a sizeable series of studies have shown that reading about different conceptual modalities (e.g., auditory 'loud bell' ...

\footnotetext{
${ }^{4}$ Lynott and Connell's main purpose was to validate new linguistic materials, i.e., modality norms.
} 
visual 'pink background') incurs processing costs (from Pecher et al., 2003). Importantly, this manipulation does not concern the presentation mode of the stimulus, maintained constant, but the intrinsic conceptual modality of the stimulus concepts. The conceptual modality switch has been an extremely fruitful paradigm, whose effect has been replicated numerous times (Pecher, Zeelenberg, \& Barsalou, 2004; Solomon \& Barsalou, 2004; Marques, 2006; Vermeulen, Niedenthal, \& Luminet, 2007; van Dantzig, Pecher, Zeelenberg, \& Barsalou, 2008; Lynott \& Connell, 2009; Ambrosi, Kalenine, Blaye, \& Bonthoux, 2011; Collins et al., 2011; Hald et al., 2011; Hald et al., 2013; Scerrati et al., 2015). Of the 12 preceding studies, only one reports a non-significant effect (Scerrati, Lugli, Nicoletti, \& Borghi, 2016). The others found the effect either in RTs or in ERPs. However, we will note that none of the three studies that measured ERPs and then RTs found a significant effect in RTs (Collins et al., 2011; Hald et al., 2011; Hald et al., 2013). When referring to this, the authors of the three papers pointed to the fact that their samples were smaller than those of the RT studies. Yet, further below we offer an alternative interpretation.

The conceptual modality switch was instrumental in demonstrating the 'embodied' share in conceptual processing. Yet, those studies may have left unaccounted an extra, nonembodied portion. In so arguing, Louwerse and Connell (2011) re-analysed one of those modality-switching studies as we described above. The authors found that a dual interplay of 'conceptual systems' could predict response times (RTs) better than any single one of them. In the current study, we address this with a new turn: we prime the RTs differently over two groups, rendering a quick group who must respond below 2,600 ms, and a selfpaced group who may respond up to $8,000 \mathrm{~ms}$. These groups are intended to 'personify,' as it were, the linguistic system and the distributional system, respectively. The reason we could not implement a within-group design is that, because there were no such precedents, the direction of a potential carryover effect was unpredictable (see Barsalou, 2016 about this gap in the literature). Participants' conceptual processing might become either more linguistic or more embodied throughout the task.

Last to note, the use of particular modalities in this paradigm — based on the popular five senses-, as opposed to any more specific ones such as colour, or more general ones such as shape, responds only to the trend within this stream of studies (cf. Binder et al., 2016). Further, we do not use olfactory or gustatory items because they lack enough items (cf. Bernabeu, Louwerse, \& Willems, in prep.; Lynott \& Connell, 2009, 2013), and they are 
not necessary for our design.

\section{General design}

The experimental design resembles a matryoshka doll in its embeddings. Each trial is made up of a property and a concept which are subsequently presented. Further, trials add up to trial pairs, allowing for a controlled transition within each trial pair. The controlled variable in these transitions is the dominant conceptual modality of each trial. For instance, a transition from auditory to visual modality might be: Melodious Voice / Tough Security. Transitions are precisely the basis of the events that are expected to trigger certain Event-Related Potentials. Finally, and most importantly, such transitions/events enable three experimental conditions: (1) modality-shifts to the embodied and linguistic conceptual systems, (2) modality-shifts to the embodied conceptual system alone, and (3) no modality-shifts. In order to realize the three conditions, three different conceptual modalities were used. Please regard modality and condition as separate, without a oneto-one correspondence: The conceptual modality applies per trial, whereas the Condition applies per event (trial+trial compound).

\begin{tabular}{cc} 
Trials: property + concept & Events (covert): trial + trial \\
\hline 216 trials: & 108 events: \\
144 Visual (property + concept) & 36 Visual - Visual \\
36 Auditory (property + concept) & 36 Haptic - Visual \\
36 Haptic (property + concept) & 36 Auditory - Visual
\end{tabular}

In each of the three conceptual modalities used, the number of true trials was virtually identical to that of false trials. The Auditory modality has 18 true and 18 false trials; the Haptic modality has 17 true versus 19 false trials; and the Visual modality has 74 true versus 70 false trials.

Crucially, transitions are covert to participants, as every trial has to be verified identically. The ERPs are measured at the onset of the first word - i.e., the property - of the second trial in each Event (see timeline figure further below). Unlike previous ERP studies within this paradigm (Hald et al., 2011, 2013; Collins et al., 2011), we measure ERPs at the first word in the target trials, rather than at the second word, due to multiple advantagesespecially, the measurement of a more current switch, the cancellation of property-toconcept lexical effects which would be confounds, and the possibility to let the Quick 
Group respond immediately after the concept without blurring the signal away with movement artefacts. Also importantly, this adjustment allowed us to have false relationships in some target trials, which otherwise would not have been possible (Hald et al., 2013). These advantages are further demonstrated in the following paragraph.

The conceptual modality switch is a highly robust effect, having survived nearly unscathed for over a decade. The replications are aplenty, as cited above. Yet, recently, Scerrati et al. (2016) failed to replicate the effect with an RT measure, either in a semantic task or in a semantic task. With smaller samples, neither has the effect been reflected in RTs, in any of the three previous ERP studies (Collins et al., 2011; Hald et al., 2011; Hald et al., 2013). The lack of an effect in RTs should not be too surprising if we consider the fact that the switch is elicited seconds before the measure. That is, the switch is expected to emerge at the onset of the first content word of the target trial-in Scerrati et al., a concept word - but the measure is being taken only after the presentation of that word, and of the following property word. Moreover, any potential, already weakened effect in said study may have been further blurred by the uncontrolled semantic distance between the property and the concept word, and furthermore by controlled processes prompted by the response to each trial.

\section{Hypotheses}

Previous ERP experiments on the conceptual modality switch measured at the second content word in the target trials, perhaps because the modality might not be encoded by participants before the second word. Indeed, in the present study, one could doubt whether the modality switch could arise fast enough to be captured in the first word - namely, in an N400, or harder yet in an 'early' N400 as in Hald et al. (2011). However, various studies did find simulation-based semantic processing that early or even earlier (Hauk \& Pulvermüller, 2004; Moscoso del Prado Martín, Hauk, \& Pulvermüller, 2006; Pulvermüller, Shtyrov, \& Ilmoniemi, 2005; Pulvermüller, Shtyrov, \& Hauk, 2009; Amsel, Urbach, \& Kutas, 2014; Van Dam, Brazil, Bekkering, \& Rueschemeyer, 2014).

The task is based on truer and falser conceptual relations. The stimuli for that are not quite natural; in fact, false trials as well as true ones contained some uncommon combinations, unbound by any of the usual collocations in language. Any such rarity might lead participants to simply attend to 'what can and cannot be said' in their language, even 
though the task requires semantic processing. Shallow processing might be thought to work against a potential modality-switching effect. Yet, on the contrary, deep semantic effects have been picked up previously through ERPs, during a lexical decision task featuring some non-words (Imbir, Spustek, \& Żygierewicz, 2016). Particularly, a Frontal N400 (FN400) and a Late Positive Component (LPC) were identified. Precisely those components were also identified as markers of modality-switching effects in the studies that precede us (Hald et al., 2011; Collins et al., 2011).

Independent variables

The present manipulation is initially based on three variables: modal transition (match/mismatch), conceptual impact of the transition (linguistic/embodied), and conceptual system group (quick/self-paced, respectively emulating linguistic and embodied 'systems'). These variables cannot be fully crossed because there are no modality mismatches that would be detected by LS but not by ES. Therefore, transition and conceptual impact are meshed into one variable, i.e., Stimulus. That leaves two independent variables, Group and Stimulus.

Group. Commonly used in conceptual processing research, property verification is a task whereby participants judge the association between a concept and a property as likely or unlikely. For example, 'a delicious pizza' would be likely, whereas 'a sweet pizza' would be unlikely. As Solomon and Barsalou (2004) found, this task can recruit both modal and amodal mental processes - and it can be difficult to tease those apart. For that reason, Collins et al. then contrived a design 'to discourage participants from shallow processing strategies relying on word association' (p. 3). In our experiment, we will rather prime both processes within different groups. This is the first experimental design, to our knowledge, featuring different groups meant to instantiate different conceptual processing mechanisms. The major dilemma is how far apart the groups can be pulled without sacrificing the validity of the comparison. Options might have included instructions to purposely imagine the associations, or not to. We opted for a more conservative option, contriving a difference as thin as possible, realized only by a different sentence in the instructions, and a different set-up in the design. For instructions, participants are told

\footnotetext{
${ }^{5}$ Incidentally, Imbir et al.'s findings seem to speak against the 'deep' and 'shallow' distinction for semantic processing. If one pole of this dichotomy yields the very effects that characterize the opposite pole, then the distinction itself might be too shallow.
} 
about the succession of property-concept pairs, and their task to judge each of them. The difference is: where one group is required to respond as fast as possible, the other is prompted to provide correct answers. Then, once in the experiment, the response limit for the quick group is $2,600 \mathrm{~ms}$, whereas for the self-paced group it is $8,000 \mathrm{~ms}$.

Stimulus. Previous studies have varied in the way properties and concepts were arranged: some had a concept then property pair (Pecher et al., 2004; Collins et al., 2011), whereas others had full sentences, i.e. property 'is a property of' concept (Solomon \& Barsalou, 2004), or concept 'can be' property (Pecher et al., 2003; Marques, 2006; Vermeulen et al., 2007; Lynott \& Connell, 2009). Quite close, Hald et al used habitual events-e.g., 'a baby drinks milk.' For the current design, we have chosen the simplest form-property then concept - for the sake of experimental control, and because none of the other options would dramatically increase the ecological validity. The critical manipulation in this is the transition across trials (e.g. 'delicious pizza' ... 'red car'). These consecutive pairs either match or mismatch in their dominant modalities. Further, they would do so differently for the two different groups, to the extent that the Quick group is rather primed for distributional processing and the Self-paced group for embodied processing. Thus, following the results from Louwerse and Connell, quite specific hypotheses may be posited. First, visual to visual transitions should constitute a modality match in both groups. Next, haptic to visual transitions would be expected to match in the Quick group but to mismatch in the Self-paced group. Finally, auditory to visual transitions would produce a mismatch in both groups.

In sum, we have a $2 * 3$ design:

$\begin{array}{lcc} & \text { Group } & \text { Transition } \\ \text { Cell 1 } & \text { Quick } & \text { Visual to visual } \\ \text { Cell 2 } & \text { Quick } & \text { Haptic to visual } \\ \text { Cell 3 } & \text { Quick } & \text { Auditory to visual } \\ \text { Cell 4 } & \text { Self-paced } & \text { Visual to visual } \\ \text { Cell 5 } & \text { Self-paced } & \text { Haptic to visual } \\ \text { Cell 6 } & \text { Self-paced } & \text { Auditory to visual }\end{array}$

Dependent variables and planned analyses

Both behavioural and electrophysiological (EEG) responses are measured. The former are RTs. The latter are ERPs. As predicted by all previous literature, RTs would be 
expected to increase with the greater violations to expectation, or specifically the greatest modality switch. The identification of ERPs will necessarily be more exploratory than confirmatory. This is because the state of affairs on linguistic ERPs would not yet allow for such an absolute specificity. Indeed, the labelling of N400 for certain components in the literature may have been stretched at times. In our particular case, what is really relevant is an expected difference in the signal, not which component that comes from. This approach is enabled by the tight control on the stimuli in the different conditions (recall that ERPs are time-locked to the property not the concept, and that trials are randomized). Luck (2005) regards such a focus on just any ERP difference as most efficient, provided a well controlled experimental design. ${ }^{6}$ That said, two potential components stand out. Most notably, the N400 or similar waves characterized by a negative deflection for greater semantic deviations (cf. Hald et al., 2011; Collins et al., 2011). This includes the frontal N400 (see also Imbir et al., 2016). Second, the P2 or close components may also be relevant (cf. van Dam et al., 2014). For greater clarity, a prediction is made explicit below with respect to a potential N400-like component, i.e., one with a deflection for the greater modality-switching effect, which coincides with the prediction for RTs. The more negative the N400 wave, the greater the RTs (Kutas \& Federmeier, 2011). Predicting a direction for less predictable components in semantic processing would be more adventurous.

Between-group analysis. The Embodied-Mismatch condition should trigger a more negative wave and greater RTs in the Quick group. In contrast, the prediction for the other conditions is fuzzier. A conceptual system hypothesis-i.e., assuming system-like, competitive processes for linguistic and embodied processing-would predict no significant differences across groups on the Total-Match and Total-Mismatch conditions. A more graded take, supporting one single process, or system, with varying realizations, would instead predict some difference across groups, with a more acute Self-paced group:

$\begin{array}{cc}\text { Transitions } & \text { Groups } \\ \text { Visual to Visual } & \text { Quick } \leq \text { Self-paced } \\ \text { Haptic to Visual } & \text { Quick }<\text { Self-paced } \\ \text { Auditory to Visual } & \text { Quick } \leq \text { Self-paced }\end{array}$

\footnotetext{
${ }^{6}$ Thanks also to Kara Federmeier for advice on this.
} 
RTs should vary according to the requirements we impose on each group, the Quick group responding significantly faster than the Self-paced group.

In contrast to the hypotheses on differences, there is an important aspect which might more likely remain unaffected by the manipulations: the latency of the ERPs. The reading of a word takes a split second. This time hardly varies under normal conditions. What is more, the sub-processes of the brain entailed in this task are temporally stable too. The primary ERP component in single-word semantic comprehension, the N400, is known for its immutable latency, and so are the particular manifestations of N400 such as the Frontal one (Kutas \& Federmeier, 2011). Even though other potentially relevant components may have more transient latencies, the stable latency of this component, which is located at the core of the word reading period, suggests that other latencies could not vary dramatically either. This fact is very relevant to setting the time windows for each or both groups. Both groups are expected to present similar latencies, and so the same windows could be applied.

Within-group analysis. The expected comparisons provided below are all quite certain, except for one (initials represent the conceptual modality: auditory, haptic or visual).

$$
\begin{array}{cc}
\text { Groups } & \text { Transitions } \\
\text { Quick } & \mathrm{V} / \mathrm{V} \leq \mathrm{H} / \mathrm{V}<\mathrm{A} / \mathrm{V} \\
\text { Self-paced } & \mathrm{V} / \mathrm{V}<\mathrm{H} / \mathrm{V} \leq \mathrm{A} / \mathrm{V}
\end{array}
$$

The contrast signalled $\mathrm{V} / \mathrm{V} \leq \mathrm{H} / \mathrm{V}$ in the quick group is subject to the issue of sufficiency of systems, especially of the linguistic system. This refers to the possibility that the linguistic system could suffice on its own in a shallow-processing condition, e.g. that in the Quick group.

Potential N400 effects. Although previous studies focused on embodied effects alone, their insights may still be relevant to us. Collins et al. (2011) investigated the alternation between visual and auditory concepts, finding a significant N400 effect for visual targets but not for the auditory ones. For the latter, they found a late positive component (LPC) instead. Critically, we only have visual items in our target trials, which will likely constrain the range of potential effects. 
In the other related study, Hald et al. (2011) set up shifts between the visual and the haptic modalities - i.e., Haptic to visual transitions - in a sentence verification task. No classical N400 was found. Yet, an N400-like effect was identified with the strongest presence in frontal regions (see window 3 in the image below, from their paper). Such a label for that effect might be more of a functional than an electrophysiological one. Yet, indeed what may be most relevant is the presence of an effect, and not its specific nature.

With a focus on the embodied system, the authors need not have controlled the depth of processing by participants. Yet now, in light of the evidence from dual accounts, it stands out that the one-second response delay applied there (an artefact-shield) may have covertly primed modality-specific simulations. In other words, such results may be underlain by a mixture of linguistic and embodied effects (Louwerse \& Connell, 2011; Santos et al., 2011).

Other potential components and artefacts. It was a challenge to go around the problem of movement artefacts produced by hand responses, especially because of the necessity that the Quick group respond as quickly as possible after the concept is presented. This was part of the reason why we chose to time-lock ERPs to the properties. Thus, timelocking to the property firstly serves to avoid artefacts stemming from the planning of the response, which would contaminate the N400. These include components produced by varying attentional demands - esp., P3b (Barber \& Carreiras, 2005; van Vliet et al., 2014; Ortu, Allan, \& Donaldson, 2013)—, and others coming from motor preparation — esp., lateralized readiness potentials (Mordkoff \& Gianaros, 2000). Further, by time-locking to the property we annuled any artefacts related to the hand movement for responding. It also let us measure ERPs in trials containing falsely associated properties and concepts, without risk of a late positive component (LPC) arising (Molinaro, Carreiras, \& Duñabeitia, 2012). Since the concept has not appeared at all by the end of the ERP signal, there cannot be any confound effects associated to semantic distance, either.

\section{Method}

Accuracy and group pretest

A behavioural pretest was conducted with 19 unpaid volunteers recruited on the Tilburg and Radboud campuses. None of them had participated in the norming of the stimuli. The purpose was to test whether the mean accuracy was above $50 \%$, and whether the self- 
paced group would respond significantly slower than the quick group. Both tests were confirmed.

\section{Participants}

The participants of the main experiment had not participated in the norming of the stimuli or in the behavioural pretest. Fifty participants were recruited from the database of the Max Planck Institute for Psycholinguistics. They all signed an informed declaration complying with the applicable ethical policies. All but two reported having fine or corrected vision and hearing. The two participants who had reported problems in the database declared no difficulties during or after the test, and both had response accuracies above $50 \%$. No participants had any neurological impairments. Participants were rewarded with a small fee for their participation. Out of all recruited participants, the first one participated only in a pilot testing. Another participant had to quit the experiment due to health problems. Yet another one was removed from the sample due to having a response accuracy below $50 \%$.

The valid, final 47 participants were randomly assigned to one of three experimental groups. The Quick group was asked to respond as quickly as possible $(n=21$; age 19-30, mean $=22.9$; seventeen females). The Self-paced group was asked to respond self-paced ( $n=21$; age $20-26$, mean $=22.3$; sixteen females). The null group received no speed constraints $(n=4 \text {; age } 20-31 \text {, mean }=25.0 \text {; all females })^{7}$

\section{Materials and design}

The stimuli, all linguistic, were made up of property and concept pairs (see 'Stimuli' file within the Supplementary material). We drew on a new norming study of conceptual modality in Dutch (Bernabeu, Louwerse, \& Willems, in prep.). The procedure for the norming was similar to that in Lynott and Connell (2009, 2013). Forty-two respondents completed a survey in which they rated the extent to which they experienced a series of properties and concepts with the senses of hearing, touch and vision. The resulting corpus, with 747 items, allowed us to select stimuli from different dominant modalities.

\footnotetext{
7 These were the first participants initially assigned to the Self-paced group. However, the written instructions they received did not explicitly request self-paced responses. As soon as we realized the negative implications of that — as participants are used to timed experiments-, those participants were relabelled as a null group. Then the instructions for the self-paced group were amended to explicitly request self-paced responses.
} 
Interestingly, this corpus presented the pattern found in the Lynott and Connell norms, with haptic and visual items closely associated, and a more isolated auditory modality.

The stimuli presented the proportions naturally found in the Dutch language, as it contained about two thirds of inflected adjectives, and one third of uninflected adjectives (Shetter, 2013). As a necessary control, no properties were repeated in either inflection form - for instance, if zwaar 'black' appeared in a trial, neither zwaar (neuter form) nor zware (masculine, feminine or plural form) would appear in any other trials.

All properties and concepts were present on the log-10 contextual diversity measure of the SUBTLEX-NL corpus - the measure best suited for the creation of experimental stimuli (Keuleers, Brysbaert, \& New, 2010).

Examples of true trials include 'Black Concealment,' 'Tender Contact,' 'Freezing Contact.' False trials included 'Dead Deck,' 'Forked Formation,' 'Bumpy Salary.' Regarding false trials, there is one major novelty to the present design. Because we timelocked ERPs to the first word in target trials, we were able to analyze the false trials without yielding a new experimental factor.

On three separate lists — one for each modality—, true and false trials were selected on a case by case basis, with the following criteria. To start, properties and concepts were only taken that were present in the log-10 contextual diversity measurement of the SUBTLEXNL corpus (Keuleers et al., 2010). Then, the true and false pairs were selected based on a subjective judgment, helped by the co-occurrence of the words in English, in the Google American corpus.

Semantic controls are key to such a design. The rather artificial, classic design and its stimuli call for the greatest possible constraint on variation across conditions. In the preceding modality-switching ERP studies, aspects such as word category, length in letters and corpus frequency of target words were matched across transition conditions up to the centesimal level. The same was done herein. Further, the current design contained a larger number of controls for the analysis of the RTs (the detailed figures are available in Appendix 1). 
Frequency, letters, and word category

The ERP-target words were tightly matched on the same three criteria as Hald et al. (2011) had matched their targets. The first of those was semantic category: all targets were properties. All but one of those belonged to the word class of adjectives, the odd one being a noun (miniatuur 'miniature'). Secondly, frequency was controlled based on the log-10 contextual diversity measure of the SUBTLEX-NL corpus $\left(M_{\text {Total-Match }}=1.92 ; M_{\text {Embodied- }}\right.$ Mismatch $\left.=1.92 ; M_{\text {Total-Mismatch }}=1.92\right)$. We used this particular measure, instead of the customary word (lemma) frequency, because it performs better at predicting RTs, and hence is better suited for the creation of stimuli (Keuleers et al., 2010). Frequencies for inflected and uninflected forms were distinguished (see Lau, Namyst, Fogel, \& Delgado, 2016, for renewed evidence of the importance of word frequency). Last to control was word length, in letters $\left(M_{\text {Total-Match }}=7.08 ; M_{\text {Embodied-Mismatch }}=7.08 ; M_{\text {Total-Mismatch }}=7.08\right)$, where a maximum of 12 letters was also maintained.

\section{Randomization}

Trials were pseudo-randomized within participant, controlling only that each context trial was followed by a target trial that had been assigned to the same condition (e.g., a context, haptic-to-visual trial would always be followed by a target, haptic-to-visual trial). As an illustration, one auditory-to-visual switch for one participant was: Soundless Answer | Bumpy Wage (bold added to ERP-target word). For another participant, the latter target trial was instead preceded by the context trial Loud Welcome. The pseudo-randomization allowed us to have the same number of trials in each condition, and also to match the target words across conditions on the criteria of corpus frequency and word length.

\section{Procedure}

By means of written instructions, participants were asked to respond in each trial whether the first word, a property, could be used to describe the second word, a concept. An example was provided based on the property 'gray' and the concept 'snow.' Snow is often white, but it can also be gray. By contrast, a property that would not match is 'pink.' Then, the instructions diverged for the different groups of participants: while the quick group was asked to respond as quickly as possible in every trial, the self-paced group was asked to respond self-paced, and the null group was unconstrained. Next, the instructions advanced that feedback would be provided for each trial. As a white lie, the instructions 
mentioned that the feedback was based on all preceding answers, and therefore participants need not worry too much about negative feedback. Last, the instructions mentioned the high sensitivity of the EEG signal, and thus participants were asked to move or blink as little as possible, and do so only while the cross was on the screen. The sensitivity of the signal was next demonstrated to the participant. They could then ask any doubts.

Participants were next presented with twelve practice trials, after which they could check doubts again. They were then told that the experiment would have a break in the middle, and the experiment started. The software Presentation was used (Neurobehavioral Systems $\left.^{\mathrm{TM}}\right)$.

Trials started with a fixation cross presented at the center of the screen for $1 \mathrm{~s}$. After 350 ms, the first word was presented for $200 \mathrm{~ms}$. An inter-stimulus blank ensued for 1,000, or $1,050 \mathrm{~ms}$, or 1,100 ms, at random, in order to ward off preparatory processes. The second word appeared then for $200 \mathrm{~ms}$. Participants could then respond. If the response provided was correct, number 1 was shown. If it was wrong, 0 was shown (this was explained and practised with the participants). If they did not respond, 'Too late' was shown. Then the next trial started. An overview of the experimental design is provided in Figure 1.

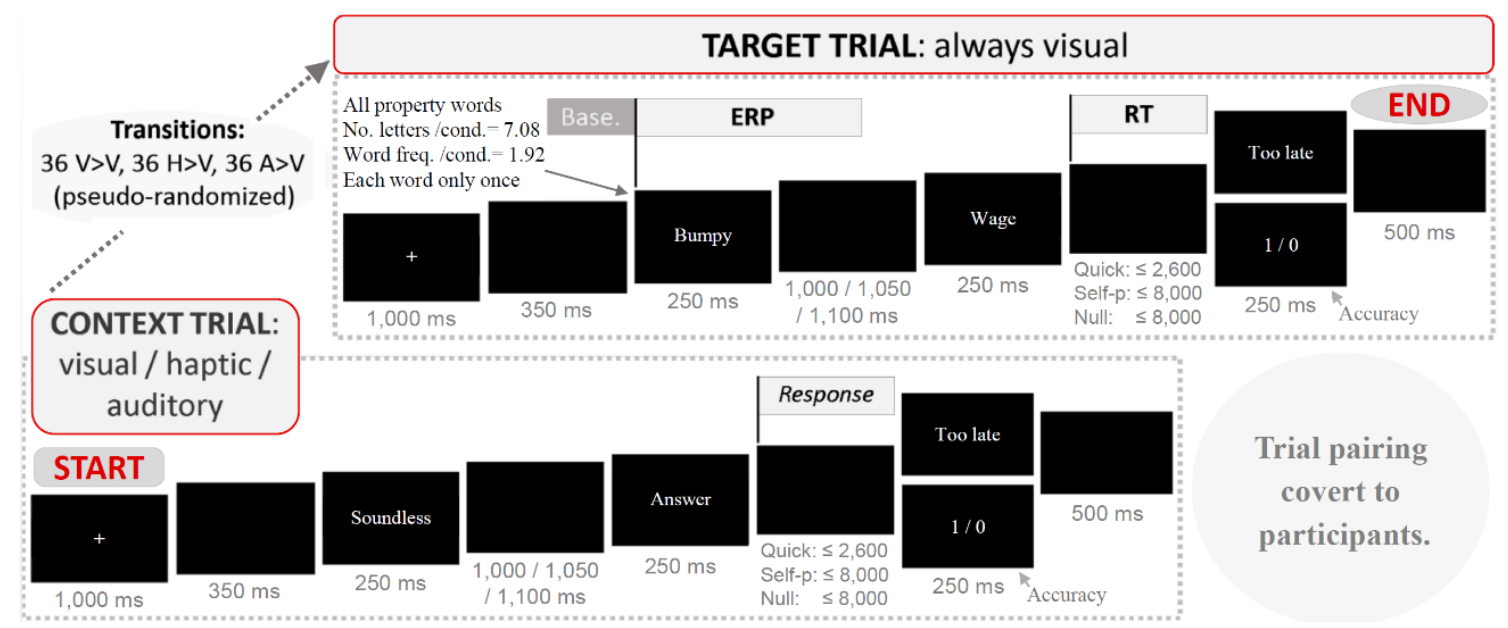

Figure 1. Overview of the experimental design. $\mathrm{A}=$ auditory, $\mathrm{H}=$ haptic, $\mathrm{V}=$ visual.

\section{EEG recording}

The EEG signal was recorded with BrainVision Recorder $1^{\mathrm{TM}}$, utilizing sixty-five active $\mathrm{Ag} / \mathrm{AgCI}$ electrodes. It was done in differential mode, whereby the online reference was set at the left mastoid, then the signal was re-referenced off-line to the right mastoid. The 
ground electrode was positioned just above the nose, at the glabella. Three other electrodes were used to register eye movements - most notably, blinks. Two of these electrodes were placed at the outer canthi of each eye to register horizontal movements, and the other electrode was placed below the left eye to register vertical movements. The remaining fifty-nine electrodes were mounted in a custom, equidistant ActiCap cap, analogous to the 10-20 disposition (see Supplementary material). Impedance was kept below $10 \mathrm{k} \Omega$, for which electrolyte gel was applied between the scalp and each electrode. The signal was amplified through BrainAmp DC amplifiers with a bandpass filter of 0.016-100 Hz, and an online sampling frequency of $500 \mathrm{~Hz}$ (i.e., a measure every $2 \mathrm{~ms}$ ).

\section{ERP preprocessing}

The signal was preprocessed in BrainVision Analyzer $2^{\mathrm{TM}}$, with the following steps: condition segmentation, automatic ocular correction (as built-in, following Gratton, Coles, \& Donchin, 1983), baseline correction, artefact rejection via semi-automatic segment selection, and averaging per switch condition (following Kappenman, Gamble, \& Luck, 2011). ${ }^{8}$ After the latter artefact rejection, the portion of segments (i.e., trials) retained was: $77.4 \%$ in the visual-to-visual condition, $78.0 \%$ in the haptic-to-visual condition, and $78.6 \%$ in the auditory-to-visual condition.

\section{ERP analysis}

Division anterior-posterior is done as in the most related previous study (Hald et al., 2011), and is supported more generally by neuroimaging (Binder, Desai, Graves, \& Conant, 2009). The division is of course very rough, but works fine with the experimental paradigm used.

We selected the time windows in an objective way, rather than based on the results (for the importance of this, see Luck \& Gaspelin, in press). Four time windows were selected based on the following criteria: (1) the most related study (Hald et al., 2011), (2) other

\footnotetext{
${ }^{8}$ The criteria for the segment selection were as follows. The critical period spanned from $300 \mathrm{~ms}$ before target onset up to $800 \mathrm{~ms}$ after target onset (the period before onset is $100 \mathrm{~ms}$ longer than the general baseline of the ERPs because that improved the selection of segments). Gradient: $75 \mu \mathrm{V} / \mathrm{ms}$. Threshold for difference between maximum and minimum voltage in segment: $\pm 150 \mu \mathrm{V}$ (this was increased or decreased by up to $40 \mu \mathrm{V}$ in a minority of cases where the automatic selection yielded too noisy waveforms), interval length $200 \mathrm{~ms}$. Amplitude: $-100 \mu \mathrm{V},+100 \mu \mathrm{V}$. Low activity: $0.5 \mu \mathrm{V}$, interval length $50 \mathrm{~ms}$. All of these criteria and their results are reflected in history files which may be consulted from the Supplementary Material.
} 
literature on language comprehension, (3) visual inspection of our waveforms. ${ }^{9}$ As a result, we only introduced a minor change with respect to the Hald et al. study, namely, we extended the latest window up to $750 \mathrm{~ms}$ post-word onset, instead of $700 \mathrm{~ms}$. This change was supported by other literature and by our waveforms (De Grauwe, Swain, Holcomb, Ditman, \& Kuperberg, 2010; Laszlo \& Federmeier, 2009). Thus, the windows were: (1) 160-216 ms, (2) 270-370 ms, (3) 350-550 ms, (4) 500-750 ms. The overlapping of some windows could be questioned, but we understand that it is the right choice because ERPs to word reading are quite variable, and also, the different stages of semantic processing likely overlap (Hauk et al., 2012; Hauk, 2016). All significance analyses were performed in R (R Core Team, 2015).

\section{Results}

We will first report on the accuracy of responses, as a basic index of the validity of the task. All participants but one responded correctly to more than half of the trials (i.e., over $50 \%$ accuracy). The only participant with a lower score had a 37\% accuracy. We decided to remove this participant. In other experiments, higher thresholds were applied, but we set $50 \%$ because the conceptual relationships in our trials were quite fuzzy. Another participant was removed from the data due to insufficient valid EEG data. The final mean accuracy without the removed participants was: in the quick group, $62 \%(\mathrm{SD}=48 \mathrm{pp})$; in the self-paced group, 63\% ( $\mathrm{SD}=48 \mathrm{pp})$; and in the null group, 64\% $(\mathrm{SD}=48 \mathrm{pp})$. The Quick and the Self-paced groups differed in their speeds by $200 \mathrm{~ms}$. This mean difference was hardly noticeable, however, due to large variation within both groups.

\section{ERPs}

Data from one participant had to be discarded from the statistical analyses due to insufficient valid data. Only 1, 2, and 4 trials were preserved per condition, out of the original 36 trials per condition.

The ERP results revealed a noticeable effect of modality transition from early on in word recognition, through the course of word processing. The effect was elicited by both types of switch relative to the non-switch, and influenced both two groups within anterior and posterior sites. The effect did start slightly earlier in the Quick group. It was also slightly

\footnotetext{
${ }^{9}$ The time windows in the Hald et al. (2011) study were: $160-215 \mathrm{~ms}, 270-370 \mathrm{~ms}, 350-550 \mathrm{~ms}$, and 500$700 \mathrm{~ms}$ post word onset.
} 
stronger overall in the Self-paced group, and in posterior rather than anterior sites. Figure 2 presents difference topographies (with time windows that are different from those set for the analysis below). Figure 3 then presents the waveforms (note that an interactive view of the waveforms per group and electrode is also available in the Supplementary material).

Mixed effects models were used to statistically analyse the results (Tables 1 and 2). These models allow for well-powered analyses while maintaining a conservative approach, particularly because variation within participants is controlled for (Baayen, Davidson, \& Bates, 2008). The models were fitted maximally, including any possible random and fixed effects that may sensibly obtain (Barr, Levy, Scheepers, \& Tily, 2013). Significance was tested from a chi-square performed on likelihood ratio test for each new factor tried on the model. With the current sample, this method is adequate (Luke, 2016). The fit of all mixed effects models was high, ranging from .711 to .866. The fit of the follow-up ANOVAs was high for windows 1 to 3, and medium for window 4 (Cohen, 1988).

Significance is large in all windows, likely due in part to the amount of data. This does not question the validity of the analyses, however, because there are important, nonsignificant effects, such as Group in the fourth window, and the interaction of Modality Transition and Group in all but the first window.

The most remarkable result, besides the broad, widespread effect, is the emergence of it in the first time window, a finding that is unprecedented in the conceptual modality switch paradigm (Figure 4). Granted, only three studies had measured ERPs, and none had timelocked to the first content word in the target trial. This result is at one with recent studies in suggesting that word comprehension-often used for studying conceptual processing - is an even quicker process than we used to think. Context seems to become available as soon as a word is recognized, that is, within the first $200 \mathrm{~ms}$ (see also, van Dam, Brazil, Bekkering, \& Rueschemeyer; cf. Sereno, Brewer, \& O’Donnell, 2003). Accordingly, we need to turn to measures of high temporal precision to study conceptual processing. 


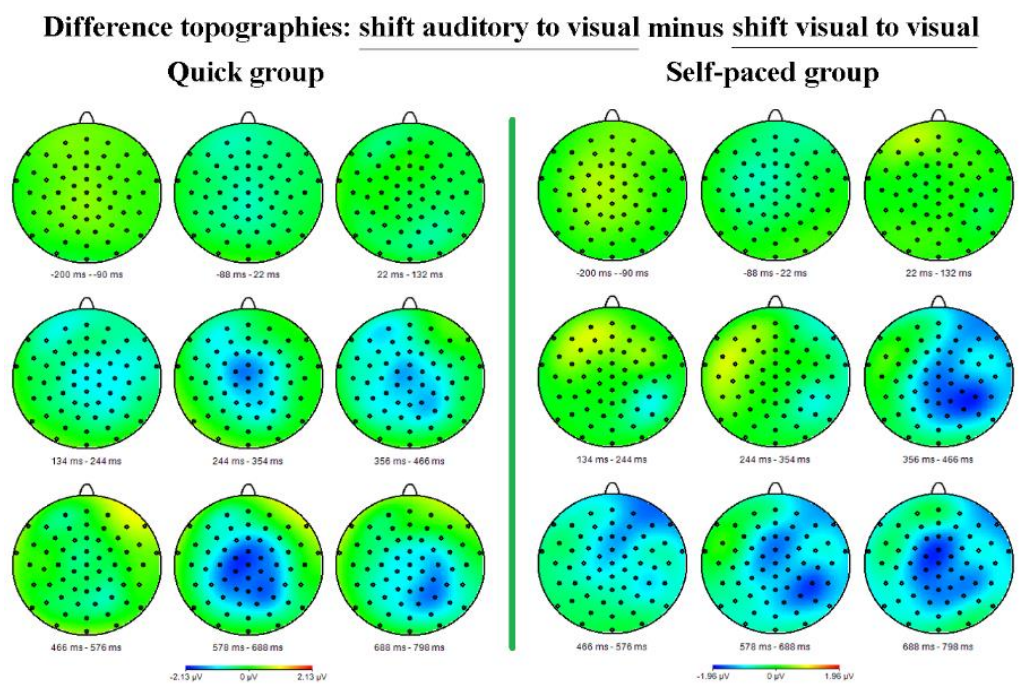

Difference topographies: shift auditory to visual minus shift haptic to visual Quick group Self-paced group

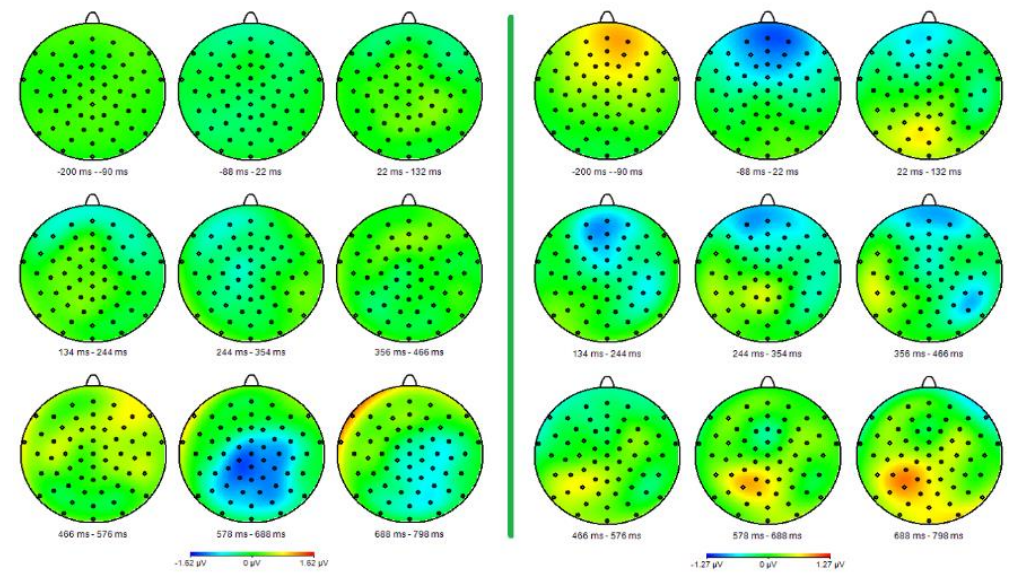

Difference topographies: shift haptic to visual minus shift visual to visual

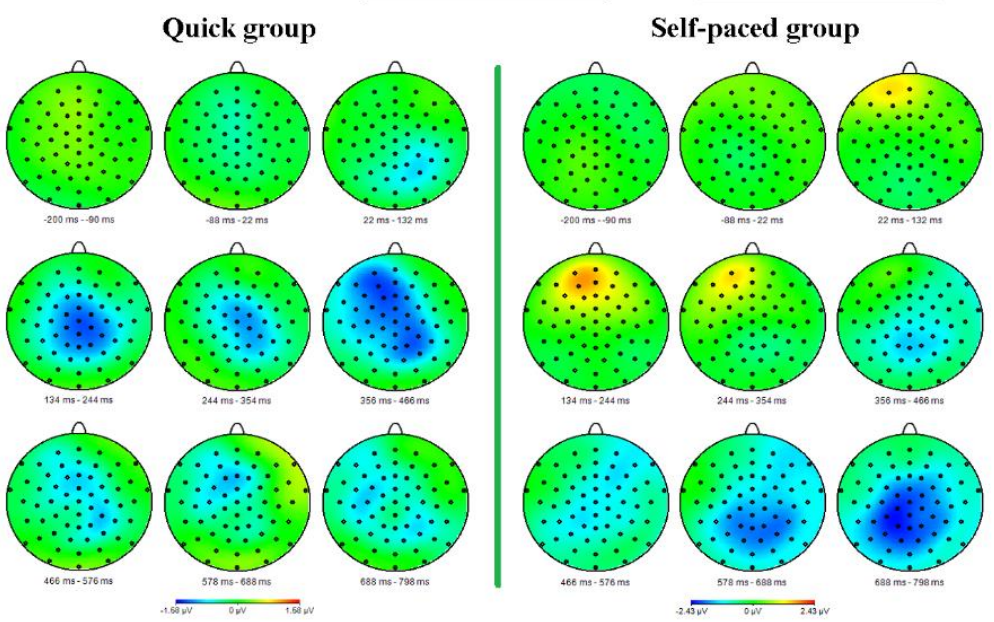

Figure 2. Subtraction topographies for the weaker and the stronger switch in both groups. 
Quick group, anterior electrodes

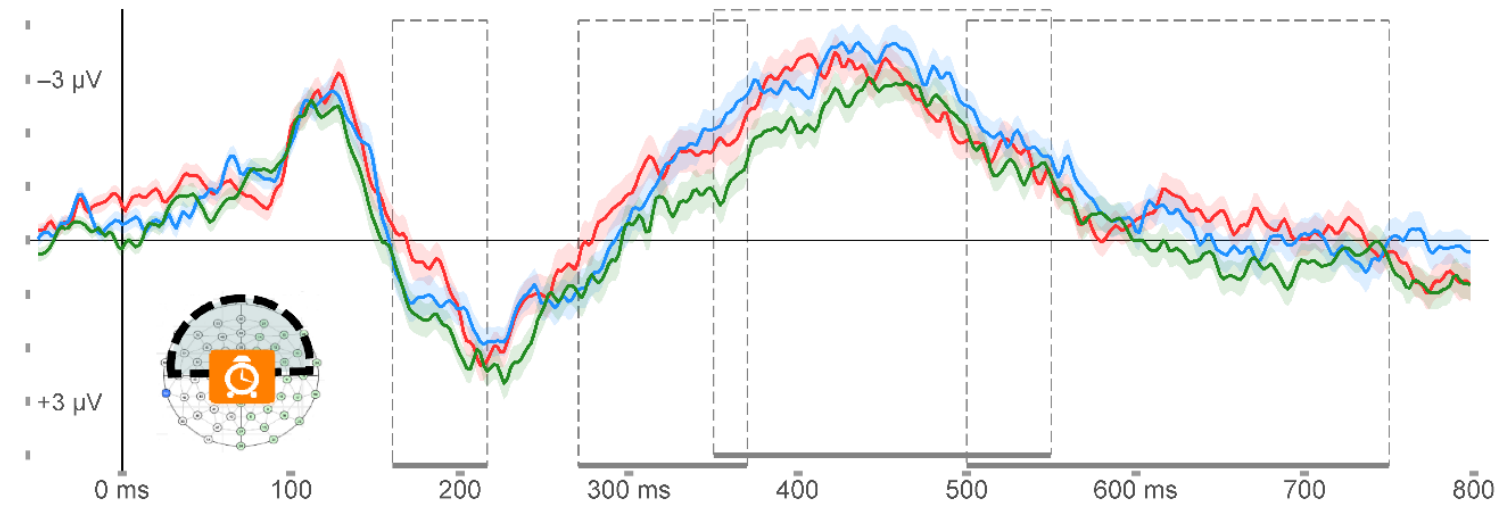

Quick group, posterior electrodes

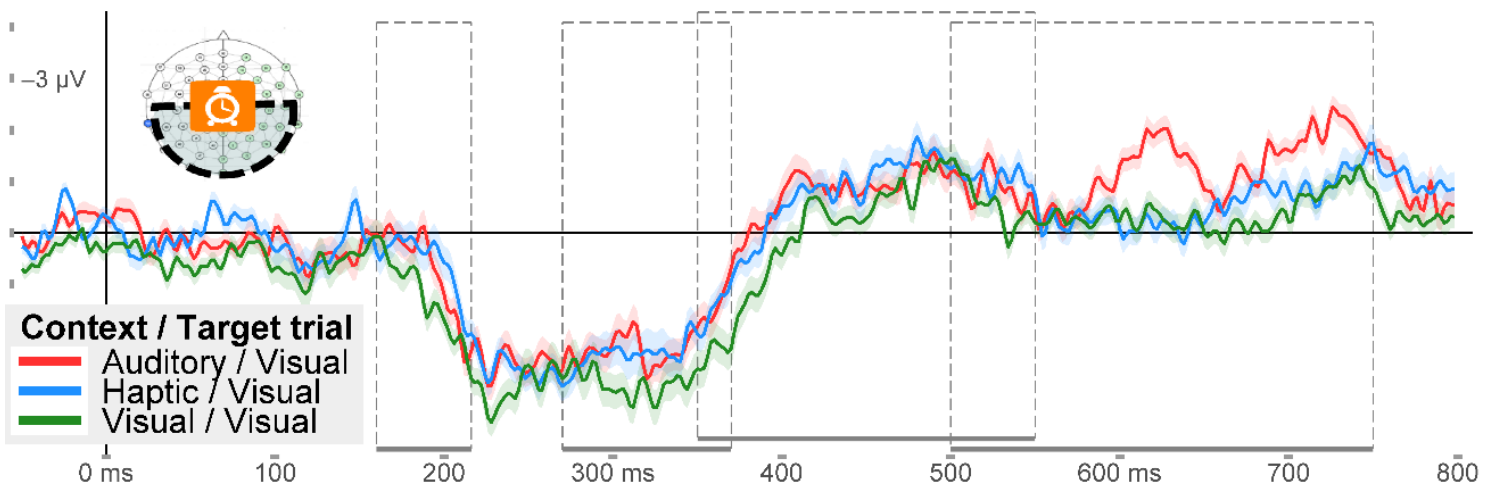

Self-paced group, anterior electrodes

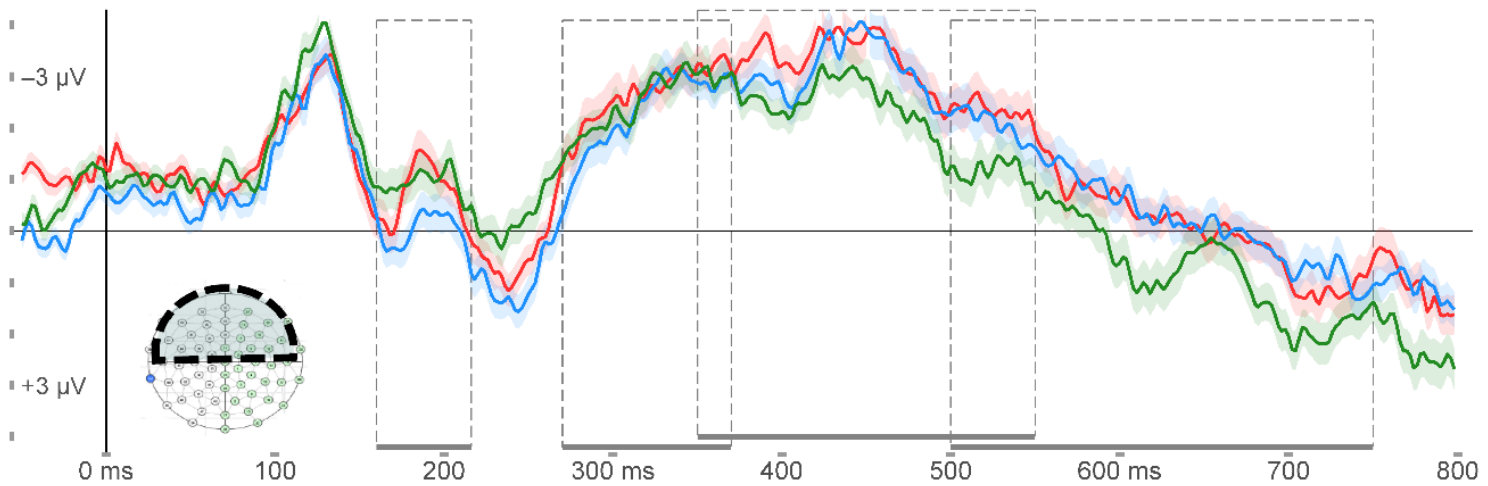

Self-paced group, posterior electrodes

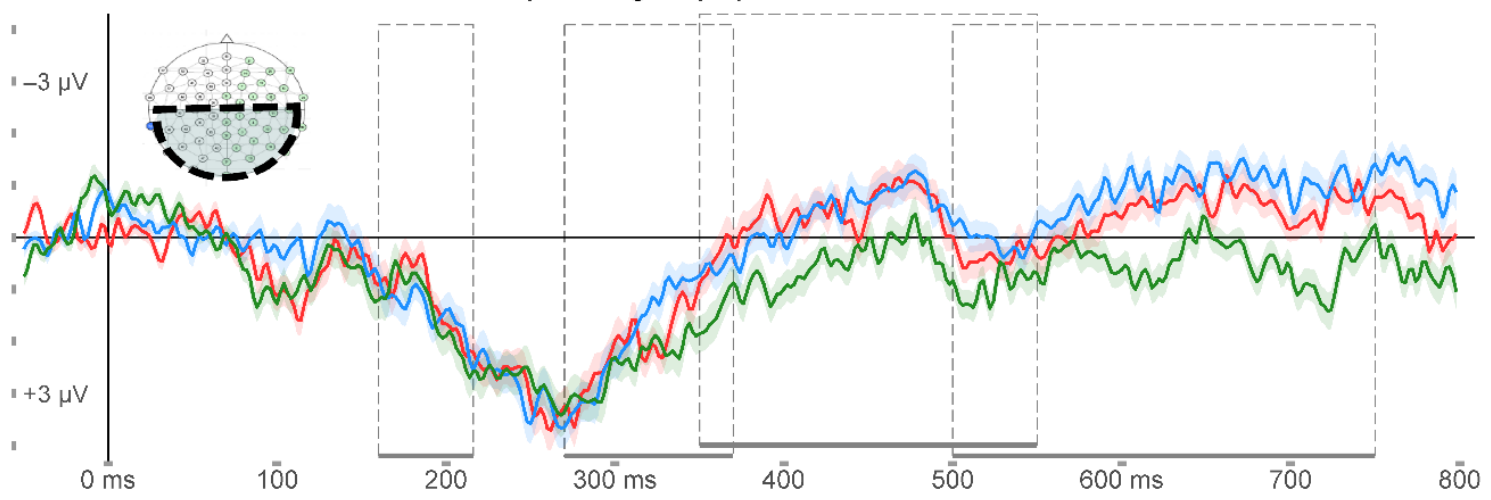

Figure 3. Waveforms. Negative plotted upward. 95\% confidence intervals per time point, and time windows are displayed. 


\begin{tabular}{|c|c|}
\hline Window & Final model \\
\hline W1 (160-216 ms) & $\begin{array}{l}\text { Imer(microvolts }(1 \mid \text { participant })+\text { time+ }(1 \mid \text { participant:time })+ \\
\text { (1| electrode })+(1 \mid \text { electrode:participant })+(1 \mid \text { condition: } \\
\text { participant })+(1 \mid \text { electrode: participant: condition })+\text { location } \\
\text { + Lefthanded + location : condition + location: group + } \\
\text { condition }: \text { s_Age_months + condition: group: location, } \\
\text { data=EEG.window1[!EEG.window1\$group=='null',], } \\
\text { control = lmerControl }(\text { optCtrl=list }(\operatorname{maxfun=10000000))})\end{array}$ \\
\hline W2 (270-370 ms) & $\begin{array}{l}\text { Imer(microvolts } \sim(1 \mid \text { participant })+\text { time+ }(1 \mid \text { participant:time })+ \\
\\
\quad(1 \mid \text { electrode })+(1 \mid \text { electrode:participant })+(1 \mid \text { condition: } \\
\text { participant })+(1 \mid \text { electrode: participant: condition })+\text { location } \\
+ \text { condition + location:condition + group : location, } \\
\text { data=EEG.window2[!EEG.window2\$group=='null',], } \\
\text { control = lmerControl }(\text { optCtrl=list }(\operatorname{maxfun}=10000000)))\end{array}$ \\
\hline W3 (350-550 ms) & $\begin{aligned} \text { lmer(microvolts } ~ & (1 \mid \text { participant })+\text { time+ }(1 \mid \text { participant: time }) \\
& +(1 \mid \text { electrode })+(1 \mid \text { electrode: participant })+(1 \mid \text { condition: } \\
& \text { participant })+(1 \mid \text { group: time })+(1 \mid \text { electrode: participant: } \\
& \text { condition })+ \text { location+ condition }+ \text { group : location, } \\
& \text { data=EEG.window3[!EEG.window3\$group=='null',], } \\
& \text { control = lmerControl }(\text { optCtrl=list }(\operatorname{maxfun}=10000000)))\end{aligned}$ \\
\hline W4 (500-750 ms) & $\begin{array}{l}\text { lmer(microvolts } \sim(1 \mid \text { participant })+\text { time+ }(1 \mid \text { participant: time })+ \\
\text { (1|electrode })+(1 \mid \text { electrode: participant })+(1 \mid \text { condition: } \\
\text { participant })+(1 \mid \text { group: time })+(1 \mid \text { electrode: participant: } \\
\text { condition })+ \text { s_Age_months + condition, } \\
\text { data=EEG.window4, } \\
\text { control = lmerControl }(\text { optCtrl=list }(\operatorname{maxfun}=10000000)))\end{array}$ \\
\hline
\end{tabular}

Table 1. Final models, coded and shown here in R language (R Core Team, 2015). Key: (1| random slopes $)+(1 \mid$ random slopes : by another variable $)+$ fixed effect.

\begin{tabular}{|c|c|c|c|c|c|}
\hline \multicolumn{6}{|c|}{ Critical results $^{*}$} \\
\hline Data & Variable & Levels or contrasts & Estimate & Statistical test** & $R^{2}$ \\
\hline \multirow{10}{*}{ All W1 } & \multirow{2}{*}{ Location } & Anterior & Intercept & \multirow{2}{*}{$\chi^{2}(1, N=46)=10.50, p=.001$} & \multirow{10}{*}{.800} \\
\hline & & Posterior & -0.26 & & \\
\hline & \multirow{3}{*}{$\begin{array}{l}\text { Modality } \\
\text { transition }\end{array}$} & (1) Visual / Visual & NA & \multirow{3}{*}{$\chi^{2}(2, N=46)=1.40, p=.498$} & \\
\hline & & (2) Haptic / Visual & NA & & \\
\hline & & (3) Auditory / Visual & NA & & \\
\hline & \multirow{2}{*}{ Group } & Quick & NA & \multirow{2}{*}{$\chi^{2}(1, N=42)=1.57, p=.210$} & \\
\hline & & Self-paced & NA & & \\
\hline & $\begin{array}{l}\text { Transition } \\
\text { x Location }\end{array}$ & - & - & $\chi^{2}(4, N=42)=71.15, p<.001$ & \\
\hline & $\begin{array}{l}\text { Transition } \\
\text { x Group }\end{array}$ & - & NA & $\chi^{2}(2, N=42)=3.31, p=.191$ & \\
\hline & $\begin{array}{l}\text { Transition } \\
\text { x Location }\end{array}$ & - & - & $\chi^{2}(4, N=42)=26.85, p<.001$ & \\
\hline
\end{tabular}




\begin{tabular}{|c|c|c|c|c|c|}
\hline & x Group & & & & \\
\hline \multirow{2}{*}{$\begin{array}{l}\text { W1: Quick, } \\
\text { anterior }\end{array}$} & \multirow{2}{*}{$\begin{array}{l}\text { Transition } \\
\text { contrasts }\end{array}$} & $(1)$ versus $(2 \& 3)$ & 0.18 & $\begin{array}{l}F(54,13049)=258.90 \\
t=11.04, p<.001\end{array}$ & \multirow{2}{*}{.515} \\
\hline & & (2) versus (3) & -0.14 & $t=-4.98, p<.001$ & \\
\hline \multirow{2}{*}{$\begin{array}{l}\text { W1: Quick, } \\
\text { posterior }\end{array}$} & \multirow{2}{*}{$\begin{array}{l}\text { Transition } \\
\text { contrasts }\end{array}$} & (1) versus $(2 \& 3)$ & 0.20 & $\begin{array}{l}F(54,13049)=127.90 \\
t=11.69, p<.001\end{array}$ & \multirow[t]{2}{*}{.343} \\
\hline & & (2) versus (3) & 0.06 & $t=2.09, p=.074$ & \\
\hline \multirow{2}{*}{$\begin{array}{l}\text { W1: } \\
\text { Self-paced, } \\
\text { anterior }\end{array}$} & \multirow{2}{*}{$\begin{array}{l}\text { Transition } \\
\text { contrasts }\end{array}$} & (1) versus $(2 \& 3)$ & -0.19 & $\begin{array}{l}F(54,13049)=307.20 \\
t=-10.81, p<.001\end{array}$ & \multirow[t]{2}{*}{.558} \\
\hline & & (2) versus (3) & -0.34 & $t=-11.25, p<.001$ & \\
\hline \multirow{2}{*}{$\begin{array}{l}\text { W1: } \\
\text { Self-paced, } \\
\text { posterior }\end{array}$} & \multirow{2}{*}{$\begin{array}{l}\text { Transition } \\
\text { contrasts }\end{array}$} & (1) versus $(2 \& 3)$ & 0.05 & $\begin{array}{l}F(54,13049)=109.70 \\
t=2.21, p=.054\end{array}$ & \multirow[t]{2}{*}{.309} \\
\hline & & (2) versus (3) & -0.10 & $t=-2.73, p=.012$ & \\
\hline \multirow{10}{*}{ All W2 } & \multirow{2}{*}{ Location } & Anterior & Intercept & \multirow{2}{*}{$\chi^{2}(1, N=46)=83.91, p<.001$} & \multirow{10}{*}{.866} \\
\hline & & Posterior & 3.43 & & \\
\hline & \multirow{3}{*}{$\begin{array}{l}\text { Modality } \\
\text { transition }\end{array}$} & (1) Visual / Visual & Intercept & \multirow{3}{*}{$\chi^{2}(2, N=46)=6.40, p=.041$} & \\
\hline & & (2) Haptic / Visual & -0.25 & & \\
\hline & & (3) Auditory / Visual & -0.48 & & \\
\hline & \multirow{2}{*}{ Group } & Quick & NA & \multirow{2}{*}{$\chi^{2}(1, N=42)=1.88, p=.170$} & \\
\hline & & Self-paced & NA & & \\
\hline & $\begin{array}{l}\text { Transition } \\
\mathrm{x} \text { Location }\end{array}$ & - & - & $\chi^{2}(2, N=46)=10.89, p=.004$ & \\
\hline & $\begin{array}{l}\text { Transition } \\
\text { x Group }\end{array}$ & - & NA & $\chi^{2}(2, N=42)=1.35, p=.509$ & \\
\hline & $\begin{array}{l}\text { Transition } \\
\text { x Location } \\
\text { x Group }\end{array}$ & - & - & $\chi^{2}(4, N=42)=28.16, p<.001$ & \\
\hline \multirow{2}{*}{$\begin{array}{l}\text { W2: Quick, } \\
\text { anterior }\end{array}$} & \multirow{2}{*}{$\begin{array}{l}\text { Transition } \\
\text { contrasts }\end{array}$} & (1) versus $(2 \& 3)$ & 0.26 & $\begin{array}{l}F(59,21234)=667.20 \\
t=19.62, p<.001\end{array}$ & \multirow[t]{2}{*}{.649} \\
\hline & & (2) versus (3) & -0.06 & $t=-2.75, p=.012$ & \\
\hline \multirow{2}{*}{$\begin{array}{l}\text { W2: Quick, } \\
\text { posterior }\end{array}$} & \multirow{2}{*}{$\begin{array}{l}\text { Transition } \\
\text { contrasts }\end{array}$} & (1) versus $(2 \& 3)$ & 0.23 & $\begin{array}{l}F(59,21234)=244.80 \\
t=15.21, p<.001\end{array}$ & \multirow[t]{2}{*}{.403} \\
\hline & & (2) versus (3) & -0.04 & $t=-1.53, p=.251$ & \\
\hline \multirow{2}{*}{$\begin{array}{l}\text { W2: } \\
\text { Self-paced, } \\
\text { anterior }\end{array}$} & \multirow{2}{*}{$\begin{array}{l}\text { Transition } \\
\text { contrasts }\end{array}$} & $(1)$ versus $(2 \& 3)$ & -0.01 & $\begin{array}{l}F(59,21234)=427.00 \\
t=-0.90, p=.737 \\
\end{array}$ & \multirow[t]{2}{*}{.541} \\
\hline & & (2) versus (3) & 0.17 & $t=-6.58, p<.001$ & \\
\hline $\begin{array}{l}\text { W2: } \\
\text { Self-paced, }\end{array}$ & Transition & (1) versus $(2 \& 3)$ & 0.18 & $\begin{array}{l}F(59,21234)=290.10, \\
t=11.64, p<.001\end{array}$ & .445 \\
\hline & & (2) versus (3) & 0.06 & $t=2.12, p=.068$ & \\
\hline & Iocation & Anterior & Intercept & $\gamma^{2}(1-N-46)-6101 n<001$ & \\
\hline & Location & Posterior & 2.00 & $\chi^{2}(1, N=46)=61.01, p<.001$ & \\
\hline & & (1) Visual / Visual & Intercept & & \\
\hline All W3 & $\begin{array}{l}\text { Modality } \\
\text { transition }\end{array}$ & (2) Haptic / Visual & -0.72 & $\chi^{2}(2, N=46)=9.47, p=.017$ & .809 \\
\hline & & (3) Auditory / Visual & -0.69 & & \\
\hline & Groun & Quick & NA & $\gamma^{2}(1-N=42)=004 n=835$ & \\
\hline & Group & Self-paced & NA & $\chi^{2}(1, N=42)=0.04, p=.835$ & \\
\hline
\end{tabular}




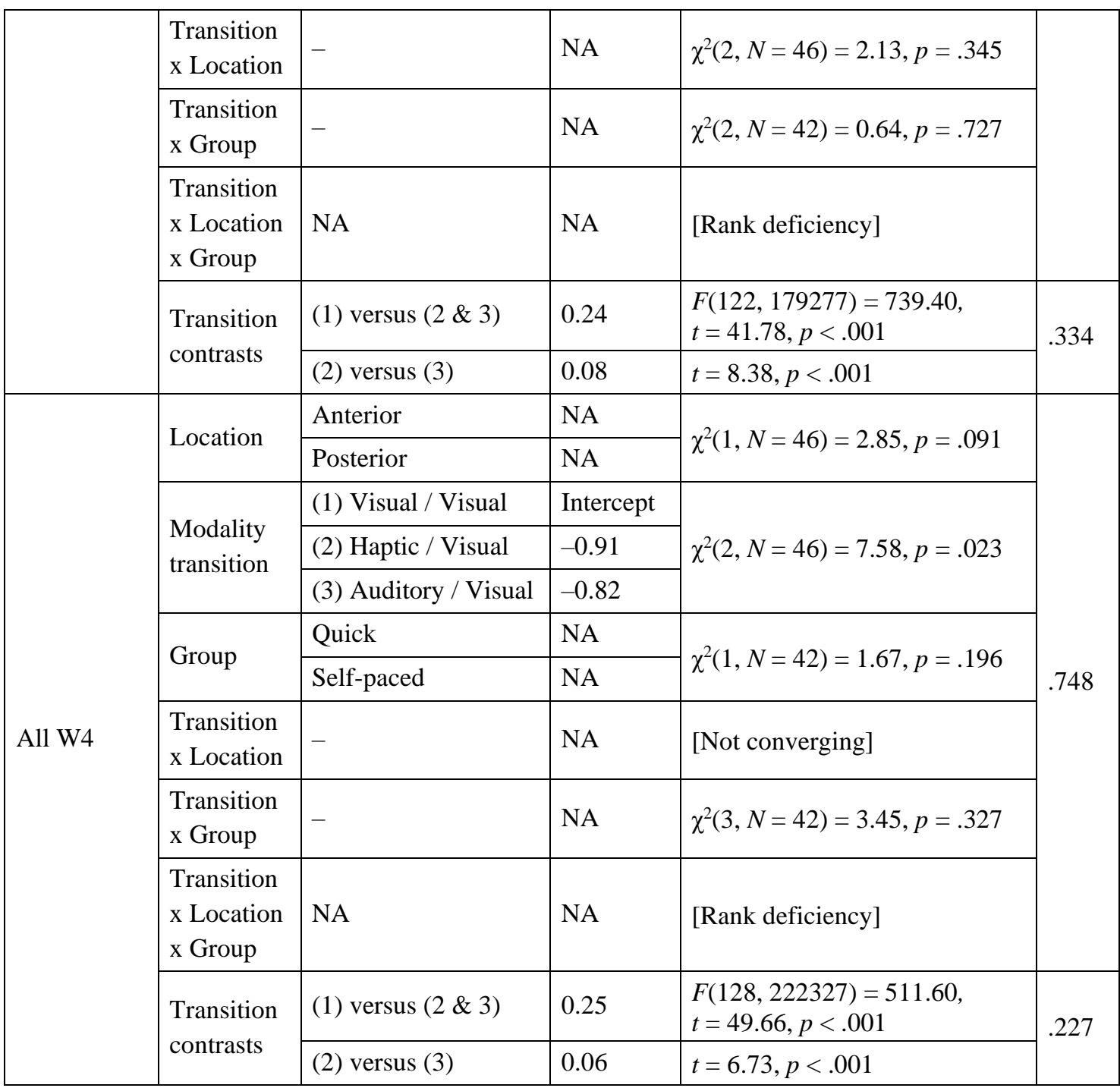

Table 2. Critical results on the ERPs: experimental variables in the mixed effects model for each window. In the third and fourth columns, hyphens appear instead of data that do exist but are already reflected in the follow-ups and in the plots. In the fifth column, follow-up ANOVAs were Bonferroni-corrected by multiplying the $p$ value by the number of tests, namely 2 (see Armstrong, 2014). In the last column, the $R^{2}$ provided for full models is of the standard, 'multiple' type, whereas the $R^{2}$ for the follow-up ANOVAs is the one 'adjusted' for the number of predictors. * For the ANOVAs, we provide the $F$ statistic only in the above contrast.

The effect then extends through the pre-N400 window to the classic N400. The N400 ERP is sensitive to a large breadth of violations to expectation, which extends from linguistic frequency linguistic semantics, and from maths (Kutas \& Federmeier, 2011) to even social interaction (Goregliad Fjaellingsdal, Ruigendijk, Scherbaum, \& Bleichner, 2016). The negativity that characterizes the effect overall might resound of the N400. However, arising in the first time window already in the Quick group, it is clear that it is not all dependent on this component. 

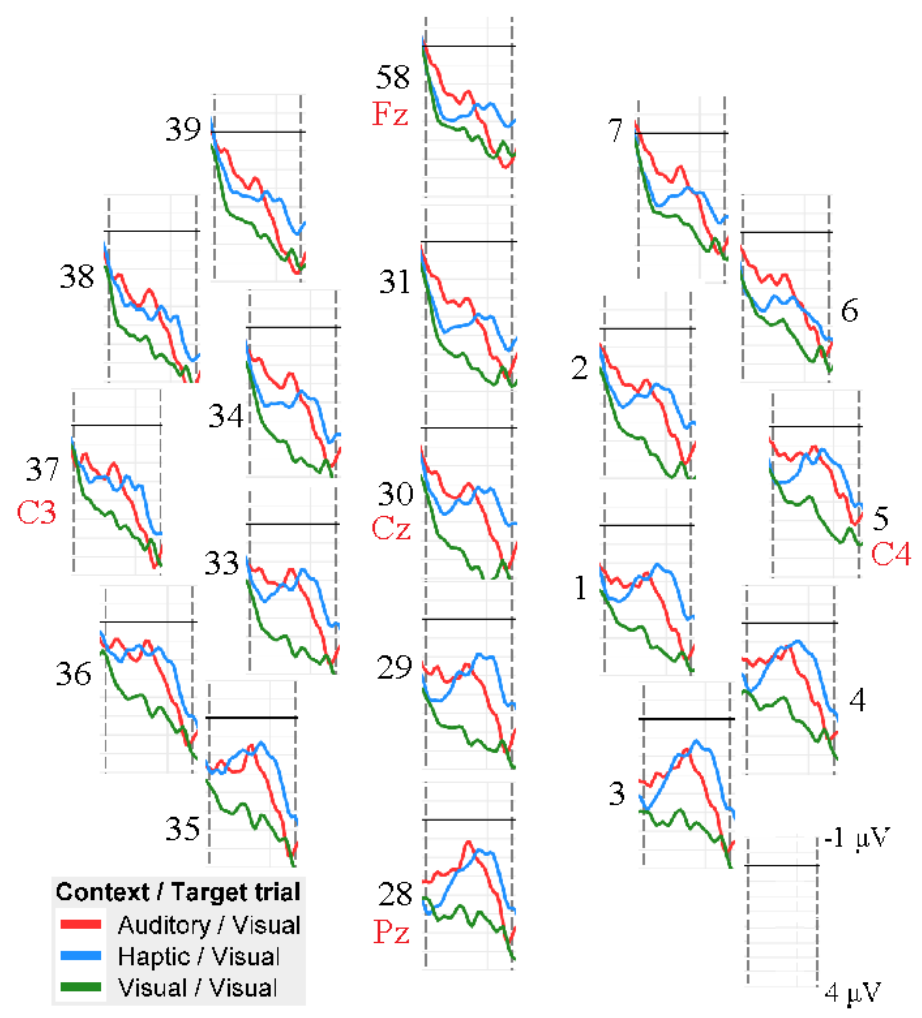

Figure 4. Close-up of the electrodes around $\mathrm{Cz}$ in the Quick group and in time window 1 . The scope of the microvolts was reduced as shown, in order to best visualize the consistency of the effect over the scalp. Red labels correspond to the international 10/20 montage.

Last, the ending window is to capture the LPC/P600. The negativity effect continues here, with a remarkable feature. Although there is no significant interaction of Group and Modality Transition, the waveforms present a pattern adjusting exactly to the predictions we made on the basis of Louwerse and Connell (2011). Whilst the quick group picks up the auditory-to-visual switch more notoriously than the haptic-to-visual switch - that is, in the posterior location-, the self-paced group picks up both switch types to quite the same extent.

The detailed hypotheses we had set for the interaction of Group and Modality Transition were not met, likely because the separation between the two groups was too narrow. The self-paced participants did not take nearly as long to respond as they were expected to.

Response times

This design was tailored to measure ERPs. RTs were not reliable enough regarding the CMS because the last word in the target trials - critical for RTs - had not been matched across conditions on the essential criteria (see Materials section above). Nonetheless, we 
analysed RTs, statistically controlling for the confounds. Any target trials falling more than 3 standard deviations away from the mean of a given participant in a given condition were considered outlier trials, and were thus removed. The removed trials represented $1.28 \%$ of all target trials. The mixed effects model applied followed the same procedure as that applied for the ERPs. The terms selected were as follows: first, random intercepts for items, for number of letters of the concept word, and for the interaction of that with each participant; second, random slopes for trial number per participant; and third, fixed effects for trial number, including quadratic and cubic trends, and for the number of letters of the concept. This model had a high explained variance, $R^{2}=.508$. The effects of Transition, Group, and the interaction thereof were not significant, all $p \mathrm{~s}>.05$.

\section{Accuracy}

The last measure to analyse is accuracy. This is the least relevant because of the nature task. The relationship between the property and the concept in each trial tended to be medium (unlike in previous studies with clearer pairings; e.g., Pecher et al., 2003). Hence our mean accuracy was rather low, 63\%. The figures, presented in Appendix 2, were not submitted to rigorous statistical analysis for three reasons. First, they did not present any remarkable differences across conditions in raw. Second, accuracy was not a significant predictor on the RTs model. Last and most generally, RTs already suggested that the behavioural data did not present a significant switch effect.

\section{Discussion}

In this paper we have reviewed the literature on conceptual processing, and presented an ERP experiment. The review suggested that the timc course of any effects is critical. It is critical for research on distributional processing as well as for evidence on embodied processing. Yet, it is slightly more so for the latter because embodiment effects tend to occur later, at a stage where semantic processing may be mixed with post-emantic processes. Scrutiny of the time course may therefore help us to advance. With regard to distributional processing, we find that its research is largely computationally-based. For this reason, we need to posit very explicit hypotheses a priori, as we have seen over the last years. In our experiment, we put both take-home messages into practice as we enhanced the well-known conceptual modality switch paradigm. As the main novelty, we time-locked ERPs to the onset of the switch manipulation. The results presented an effect 
of modality switching emerging early on in lexicosemantic processing, extending on through the rest of word processing. The virtually immediate effect upon recognition of the word suggests that sensory brain regions may have a fundamental contribution in conceptual processing.

In our experiment, we pursued the greatest temporal precision because research suggests that the time course of conceptual processing may serve as a proxy for the relevance of embodiment effects (Hauk, 2016). By contrast, more lagged measurements offer less certainty because they may allow post-semantic processes to emerge before the measure. This does not mean that those results are not valid. First, the current results have converged with many of those results, and second, those studies make most of the evidence we count on to date. What the current results do suggest is that measuring effects of embodiment as soon as they are elicited - that is, from millisecond 0 - is not in vain. It may be most efficient because it helps measure the experimental manipulations at their freshest, and also helps control the time course of the effect with greater precision. In addition, it may help reducing confounds, as in the current design. In a broader context, the sheer speed of word processing converges with the pace of event processing. Indeed, identifying participants in an event may take less than a second (Wilson, Papafragou, Bunger, \& Trueswell, 2011).

This experimental design was particularly focused on the earlier part of the embodied effect, where we pursued higher temporal precision than previously. Yet by contrast, we have much less certainty on the later part of the effect. We do not know if the larger size of the effect in windows 3 and 4, compared to earlier windows, is due to a greater perceptual simulation as the comprehension process advances. Research on single word comprehension currently presents a co-occurrence of processes in those windows (Hauk, 2016). Therefore, the increase of the effect might be due to an increasing activation of sensory systems once the core meaning of the word is being, or has been, understood (Mahon \& Caramazza, 2008). This post-core activation could entail an increased acuteness of the sense that is more relevant to a word. On a third alternative, it could be that the later engagement of sensory regions was twofold: first, it would contribute to the core of conceptual processing, and meanwhile or immediately after, it would enable physiological-behavioural responses. This third hypothesis would be compatible with the embodied cognition theory, whose main tenet is the reuse of cortex for related processes in lower and higher cognition (Pulvermüller, 1999). It also seems to be tolerated by data 
suggesting post-semantic engagement of embodiment systems (see Papeo, Vallesi, Isaja, and Rumiati, 2009), but this would have to be tested. However, it is extremely complicated to tease apart the semantic core of conceptual processing from controlled processes (yet see Whitney, Kirk, O'Sullivan, Lambon Ralph, \& Jefferies, 2012).

The compatibility of distributional and embodied systems in enabling conceptual processing has implications onto the broadest questions in cognitive science. It underscores the importance of computation, whilst at the same time it notes the relevance of approaches that are less centred on computation. That is not to say that the 'embodied' system cannot be successfully interpreted as computation (cf. Mahon \& Hickok, 2016). After all, computation is clearly powerful in explaining cognitive processes. There is an entire World represented in the brain, and such a feat does seem to require scalable connections of an abstract nature. In addition, Occam's razor invites us to posit as few frameworks as possible. That is, we would advocate for a computational explanation. The single condition that is imposed by the increasingly unequivocal evidence on modal processing is that cognitive computation may take forms that depart from the classic accounts. In accounting for modal processing, we may have to invoke a 'programming language' with less arbitrary symbols, i.e., format of representation. The amodal system, by contrast, would maintain its more arbitrary symbols. Computational approaches have been on stage for a while, with an example dating back to Pulvermüller (1999). Among the most recent samples is a study in which Hebbian learning was shown to account for supra-modal knowledge (Pulvermüller \& Garagnani, 2014). The researchers found that 'disembodied' knowledge formed at the junction of different modal areas, in a fashion resonant of converge zones in the human brain (see Binder \& Desai, 2011). With a view to the future, we may find the computational approach being implemented in more tangible terms (Adams, Wennekers, Cangelosi, Garagnani, \& Pulvermuller, 2014).

The evidence resists to select a single system, on the grounds of evidence we have reviewed and presented. There seems to be valid, if not conclusive, evidence for both modal and amodal systems, based on behavioural, neuroimaging, and computational studies (respectively for each method: Santos, Chaigneau, Simmons, \& Barsalou, 2011; Binder \& Desai, 2011; Louwerse \& Connell, 2011). Notice that the evidence for one system is provided for the other as well. That is because indeed those studies supported both systems. 


\section{Future directions}

As the field starts to integrate the sensory and motor aspects of conceptual processing, we must embrace further questions. Mahon (2015) defends concentrating on the more disembodied, or symbolic, aspects of conceptual processing. Granted, they still merit many interesting questions of their own. Humans remain the symbolic species, as unraveled in Deacon (1997). However, we need not abandon research on the embodied aspects of language altogether. First, embodied cognition may be the other half of symbolic cognition, as recent evidence has suggested (Louwerse et al., 2015). Second, the embodied side of conceptual processing demands further exploration of its own, as we have been noting. We still need to pin down its precise role. Thus, some of the questions we find interesting and promising for future research are:

- What contextual and individual factors modulate the relative engagement of distributional and embodied processes? What new methods are possible?

○ Contextual factors: comprehension goal (e.g., deeper vs shallower; Louwerse \& Hutchinson, 2012), production versus comprehension and both (cf. Skipper, Devlin, \& Lametti, 2017), mode of presentation (written vs spoken vs signed; cf. Schomers \& Pulvermüller, 2016), lone task vs communicative context, genres (e.g., comprehension of literary vs journalistic texts; cf. Hartung, Burke, Hagoort, \& Willems, 2016), first vs second languages (cf. Vukovic \& Shtyrov, 2014).

○ Individual differences: age and gender (Hutchinson \& Louwerse, 2013), tendency to mentalize (Nijhoff \& Willems, 2015), to self-reflect (Xu, 2008), and to read in detail (van den Hoven, Hartung, Burke, \& Willems, 2016), expertise (cf. Calvo-Merino, Glaser, Grèzes, Passingham, \& Haggard, 2005), literacy, reading habits, and relation between acquisition and performance (Tomasello, Garagnani, Wennekers, \& Pulvermüller, in press).

○ Teasing apart core-focused engagement from post-conceptual engagement: An experimental manipulation that might help is the presentation of a distracting stimulus or task at a critical point before postconceptual processes, e.g., at $600 \mathrm{~ms}$ after word onset. Yet, such a design would likely require plenty of pretesting before it worked. 
- Greater causality inference in neuroimaging: new techniques of measure and analysis in current development could greatly facilitate research on the current topic. These include, most immediately, the combinations of fMRI and EEG, and TMS and EEG, but further combinations are possible including the three techniques together (Peters et al., 2013; but see also a critical discussion on causality in Mannino \& Bressler, 2015).

- Can the embodied system be explained in computational terms? Budding research suggests so (Pulvermüller \& Garagnani, 2014). This is arguably practical for the 'survival' of the embodied system, if we consider the endurance and power of the computational approach to cognition.

\section{Supplementary material and acknowledgments}

All data is accessible at https://osf.io/97unm.

This study was supported by the Neurobiology of Language department at the Max Planck Institute for Psycholinguistics. Thanks also to the Experimental Psychology Society for a conference presentation grant, and to Gwilym Lockwood, Johan Weustink, Monique Flecken, Ronald Fischer, Sean Roberts, and staff at Brain Products ${ }^{\mathrm{TM}}$ for expert help. 


\section{References}

Adams, F, \& Campbell, K. (1999). Modality and abstract concepts. Behavioral and Brain Sciences, 22, 4, 610.

Adams, S. V., Wennekers, T., Cangelosi, A., Garagnani, M., \& Pulvermuller, F. (2014). Learning visualmotor Cell Assemblies for the iCub robot using a neuroanatomically grounded neural network. In 2014 IEEE Symposium on Computational Intelligence, Cognitive Algorithms, Mind, and Brain (CCMB) (pp. 1-8).

Ambrosi, S., Kalenine, S., Blaye, A., \& Bonthoux, F. (2011). Modality switching cost during property verification by 7 years of age. International Journal of Behavioral Development, 35, 1, 78-83.

Amsel, B. D., Urbach, T. P., \& Kutas, M. (2014). Empirically grounding grounded cognition: the case of color. Neuroimage, 99, 149-157.

Anderson, J. R. (1978). Arguments concerning representations for mental imagery. Psychological Review, 85, $249-77$.

Anderson, A. J., Binder, J. R., Fernandino, L., Humphries, C. J., Conant, L. I., Aguilar, M., ... \& Raizada, R. D. S. (2016). Predicting neural activity patterns associated with sentences using a neurobiologically motivated model of semantic representation. Cerebral Cortex. doi:10.1093/cercor/bhw240.

Andrews, M., Frank, S. L., \& Vigliocco, G. (2014). Reconciling embodied and distributional accounts of meaning in language. Topics in Cognitive Science, 6, 359370.

Baayen, R. H., Davidson, D. J., \& Bates, D. M. (2008). Mixed-effects modeling with crossed random effects for subjects and items. Journal of Memory and Language, $59,4,390-412$.

Barber, H., \& Carreiras, M. (2005). Grammatical gender and number agreement in Spanish: an ERP comparison. Journal of Cognitive Neuroscience, 17, 1.

Barsalou, L. W. (1999). Perceptual symbol systems. Behavioral and Brain Sciences, 22, 577-600.

Barsalou, L. W. (2016). On staying grounded and avoiding Quixotic dead ends. Psychonomic Bulletin \& Review, 23, 4, 1122-1142.

Barsalou, L. W., Santos, A., Simmons, W. K., \& Wilson, C. D. (2008). Language and simulation in conceptual processing. In M. De Vega, A. M. Glenberg, \& A. C. Graesser (Eds.), Symbols, embodiment, and meaning (pp. 245-283). Oxford: 
Oxford University Press.

Bernabeu, P., Louwerse, M. M., \& Willems, R. M. (in prep.). Modality exclusivity norms for 747 properties and concepts in Dutch: a replication of English. Retrieved from https://dx.doi.org/10.6084/m9.figshare.3459632.v37

Binder, J. R., Conant, L. L., Humphries, C. J., Fernandino, L., Simons, S. B., Aguilar, M., \& Desai, R. H. (2016). Toward a brain-based componential semantic representation. Cognitive Neuropsychology, doi:10.1080/02643294.2016.1147426

Binder, J. R., \& Desai, R. H. (2011). The neurobiology of semantic memory. Trends in Cognitive Sciences, 15, 527-536.

Binder, J. R., Desai, R. H., Graves, W. W., \& Conant, L. L. (2009). Where is the semantic system? A critical review and meta-analysis of 120 functional neuroimaging studies. Cerebral Cortex 19, 12, 2767-2796.

Buccino, G., Riggio, L., Melli, G., Binkofski, F., Gallese, V., \& Rizzolatti, G. (2005). Listening to action-related sentences modulates the activity of the motor system: A combined TMS and behavioral study. Cognitive Brain Research, 24, 355-363.

Calvo-Merino, B., Glaser, D. E., Grezes, J., Passingham, R. E., \& Haggard, P. (2005). Action observation and acquired motor skills: An fMRI study with expert dancers. Cerebral Cortex, 15, 8, 1243 - 1249.

Chatterjee, A. (2010). Disembodying cognition. Language and Cognition, 2, 79-116.

Cohen, J. (1988). Statistical power analysis for the behavioral sciences ( $2^{\text {nd }}$ ed.). Hillsdale, NJ: Lawrence Erlbaum Associates.

Cohen Priva, U. (2017). Not so fast: Fast speech correlates with lower lexical and structural information. Cognition, 160, 27-34

Collins, J., Pecher, D., Zeelenberg, R., \& Coulson, S. (2011). Modality switching in a property verification task: an ERP study of what happens when candles flicker after high heels click. Frontiers in Psychology, 2.

De Grauwe, S., Swain, A., Holcomb, P. J., Ditman, T., \& Kuperberg, G. R. (2010). Electrophysiological insights into the processing of nominal metaphors. Neuropsychologia, 48(7), 1965-1984.

Deacon, T. (1997). The Symbolic Species. The Co-evolution of Language and the Human Brain. London: Penguin Books.

Desai, R. H., Herter, T., Riccardi, N., Rorden, C., \& Fridriksson, J. (2015). Concepts within reach: Action performance predicts action language processing in stroke. Neuropsychologia 71, 217-224. 
Dove, G. O. (2014). Thinking in words: language as an embodied medium of thought. Topics in Cognitive Science, 6, 3, 371-89.

Dudschig, C., de la Vega, I., De Filippis, M., \& Kaup, B. (2014). Language and vertical space: On the automaticity of language action interconnections. Cortex, 58, 151160

Edmiston, P., \& Lupyan, G. (2017). Visual interference disrupts visual knowledge. Journal of Memory and Language, 92, 281-292.

Evans, N., \& Levinson, S. C. (2009). The myth of language universals: Language diversity and its importance for cognitive science. Behavioral and Brain Sciences, $32,429-492$.

Federmeier, K. D., \& Laszlo, S. (2009). Time for meaning: Electrophysiology provides insights into the dynamics of representation and processing in semantic memory. In B. H. Ross (Ed.), Psychology of Learning and Motivation, Volume 51 (pp 1-44). Burlington: Academic Press.

Fernandino, L., Binder, J. R., Desai, R. H., Pendl, S. L., Humphries, C. J., Gross, W. L., ... \& Seidenberg, M. S. (2015). Concept Representation Reflects Multimodal Abstraction: A Framework for Embodied Semantics. Cerebral Cortex, 26, 5, 20182034.

Fodor, J. A., \& Pylyshyn, Z. W. (1988). Connectionism and cognitive architecture: A critical analysis. Cognition, 28, $3-71$.

Garcea, F. E., Dombovy, M., \& Mahon, B. Z. (2013). Preserved tool knowledge in the context of impaired action knowledge: implications for models of semantic memory. Frontiers in Human Neuroscience, 7, 120.

Goregliad Fjaellingsdal, T., Ruigendijk, E., Scherbaum, S., \& Bleichner, M. G. (2016). The N400 effect during speaker-switch-Towards a conversational approach of measuring neural correlates of language. Frontiers in Psychology, 7, 1854.

Gratton, G., Coles, M. G., \& Donchin, E. (1983). A new method for offline removal of ocular artefact. Electroencephalography and Clinical Neurophysiology, 55, 4, 468484.

Grossman, M., Anderson, C., Khan, A., Avants, B., Elman, L., \& McCluskey, L. (2008). Impaired action knowledge in amyotrophic lateral sclerosis. Neurology, 71, 18, 1396-1401.

Hald, L. A., Hocking, I., Vernon, D., Marshall, J.-A., \& Garnham, A. (2013). Exploring modality switching effects in negated sentences: further evidence for grounded 
representations. Frontiers in Psychology, 4, 93.

Hald, L. A., Marshall, J.-A., Janssen, D. P., \& Garnham, A. (2011). Switching modalities in a sentence verification task: ERP evidence for embodied language processing. Frontiers in Psychology, 2.

Hartung, F., Burke, M., Hagoort, P., \& Willems, R. M. (2016). Taking perspective: Personal pronouns affect experiential aspects of literary reading. PLoS One,11, 5, e0154732.

Hauk, O. (2016). Only time will tell-Why temporal information is essential for our neuroscientific understanding of semantics. Psychonomic Bulletin \& Review, 23, 4, 1072-1079.

Hauk, O., Coutout, C., Holden, A., \& Chen, Y. (2012). The time-course of single-word reading: Evidence from fast behavioral and brain responses. Neuroimage, 60, 2, 1462-1477.

Hauk, O., Johnsrude, I., \& Pulvermüller, F. (2004). Somatotopic representation of action words in human motor and premotor cortex. Neuron, 41, 301-307.

Hauk, O., \& Pulvermüller, F. (2004). Neurophysiological distinction of action words in the fronto-central cortex. Human Brain Mapping, 21, 3, 191-201.

Hauk, O., Shtyrov, Y., \& Pulvermüller, F. (2008). The time course of action and actionword comprehension in the human brain as revealed by neurophysiology. Journal of Physiology, Paris, 102, 50-58.

Hutchinson, S., \& Louwerse, M. (2014). Language statistics explain the spatialnumerical association of response codes. Psychonomic Bulletin \& Review, 21, 470478.

Huth, A. G., de Heer, W., A., Griffiths, T. L., Theunissen, F. E., \& Gallant, J. L. (2016). Natural speech reveals the semantic maps that tile human cerebral cortex. Nature, $532,7600,453-458$.

Imbir, K. K., Spustek, T., \& Żygierewicz, J. (2016). Effects of valence and origin of emotions in word processing evidenced by event related potential correlates in a lexical decision task. Frontiers in Psychology, 7, 271.

Kappenman, E. S., Gamble, M. L., \& Luck, S. J. (2011). ERP Boot Camp: Data Analysis Tutorials (for use with BrainVision Analyzer-2 Software). Retrieved from http://erpinfo.org/boot-camp-2007-materials/2011-boot-camp-materials/bootcamp-tutorials/tutorials/Bootcamp_tutorials_all.pdf/view

Keuleers, E., Brysbaert, M. \& New, B. (2010). SUBTLEX-NL: A new frequency measure 
for Dutch words based on film subtitles. Behavior Research Methods, 42, 3, 643650 .

Kiefer, M., Sim, E.-J., Herrnberger, B., Grothe, J., \& Hoenig, K. (2008). The sound of concepts: Four markers for a link between auditory and conceptual brain systems. Journal of Neuroscience, 28, 12224-12230.

Kutas, M., \& Federmeier, K. D. (2011). Thirty years and counting: finding meaning in the N400 component of the event-related brain potential (ERP). Annual Review of Psychology, 62, 621-647.

Landauer, T. (1999). Latent Semantic Analysis (LSA), a disembodied learning machine, acquires human word meaning vicariously from language alone. Behavioral and Brain Sciences, 22(4), 624-625.

Laszlo, S., \& Federmeier, K. D. (2009). A beautiful day in the neighborhood: An eventrelated potential study of lexical relationships and prediction in context. Journal of Memory and Language, 61, 326-38.

Lau, E. F., Namyst, A., Fogel, A., \& Delgado, T. (2016). A Direct Comparison of N400 Effects of Predictability and Incongruity in Adjective-Noun Combination. Collabra, 2(1).

Lebois, L. A. M., Wilson-Mendenhall, C. D., \& Barsalou, L. W. (2014). Are automatic conceptual cores the gold standard of semantic processing? The context-dependence of spatial meaning in grounded congruency effects. Cognitive Science, 39, 8, 1764801.

Leonard, M. K., Baud, M. O., Sjerps, M. J., \& Chang, E. F. (2016). Perceptual restoration of masked speech in human cortex. Nature Communications, 7, 13619.

Louwerse, M. M. (2011). Symbol Interdependency in Symbolic and Embodied Cognition. Topics in Cognitive Science, 3, 2, 273-302.

Louwerse, M., \& Connell, L. (2011). A taste of words: linguistic context and perceptual simulation predict the modality of words. Cognitive Science, 35, 2, 381-98.

Louwerse, M., \& Hutchinson, S. (2012). Neurological evidence linguistic processes precede perceptual simulation in conceptual processing. Frontiers in Psychology, 3.

Louwerse, M. M., Hutchinson, S., Tillman, R., \& Recchia, G. (2015). Effect size matters: the role of language statistics and perceptual simulation in conceptual processing. Language, Cognition and Neuroscience, 30, 4, 430-447.

Louwerse, M. M., \& Jeuniaux, P. (2010). The linguistic and embodied nature of conceptual processing. Cognition, 114, 1, 96-104. 
Luck, S. J. (2005). Ten simple rules for designing ERP experiments. In T. C. Handy (Ed.), Event-related potentials: a methods handbook. Cambridge, MA: MIT Press.

Luck, S. J., \& Gaspelin, N. (in press). How to get statistically significant effects in any erp experiment (and why you shouldn't). Psychophysiology.

Luke, S. G. (2016). Evaluating significance in linear mixed-effects models in R. Behavior Research Methods. DOI:10.3758/s13428-016-0809-y.

Lund, K., \& Burgess, C. (1996). Producing high-dimensional semantic spaces from lexical co-occurrence. Behavior Research Methods, Instruments \& Computers, 28, 203-208.

Lynott, D., \& Connell, L. (2009). Modality exclusivity norms for 423 object properties. Behavior Research Methods, 41, 2, 558-564.

Lynott, D., \& Connell, L. (2013). Modality exclusivity norms for 400 nouns: The relationship between perceptual experience and surface word form. Behavior Research Methods, 45, 516-526.

Mahon, B. Z. (2015). What is embodied about cognition? Language, Cognition and Neuroscience, 30, 4, 420-429.

Mahon, B.Z., \& Caramazza, A. (2008). A critical look at the Embodied Cognition Hypothesis and a new proposal for grounding conceptual content. Journal of Physiology - Paris, 102, 59-70.

Mahon, B. Z., \& Hickok, G. (2016). Arguments about the nature of concepts: Symbols, embodiment, and beyond. Psychonomic Bulletin \& Review, 23, 941-958.

Mannino, M., \& Bressler, S. L. (2015). Foundational perspectives on causality in largescale brain networks. Physics of Life Reviews, 15, 107-123.

Marques, J. F. (2006). Specialization and semantic organization: Evidence for multiple semantics linked to sensory modalities. Memory \& Cognition, 34, 1, 60-67.

Mehu, M. (2015). The integration of emotional and symbolic components in multimodal communication. Frontiers in Psychology, 6, 961.

Molinaro, N., Carreiras, M., \& Duñabeitia, J. A. (2012). Semantic combinatorial processing of non-anomalous expressions. NeuroImage, 59, 4, 3488-3501.

Monaghan, P., \& Nazir, T. A. (2009). Modelling sensory integration and embodied cognition in a model of word recognition. In J. Mayor, N. Ruh, \& K. Plunkett (Eds.), Connectionist models of behaviour and cognition II: Proceedings of the 11th Neural Computation and Psychology Workshop, University of Oxford, UK, 16-18 
July 2008. (pp. 337-348). (Progress in neural processing; Vol. 18). SINGAPORE: WORLD SCIENTIFIC PUBL CO PTE LTD.

Mordkoff, J. T., \& Gianaros, P. J. (2000). Detecting the onset of the lateralized readiness potential: A comparison of available methods and procedures. Psychophysiology, 37(3), 347-360.

Moscoso del Prado Martín, F., Hauk, O., \& Pulvermüller, F. (2006). Category specificity in the processing of color-related and form-related words: an ERP study. Neuroimage $29,1,29-37$.

Myachykov, A., Scheepers, C., Fischer, M. H., \& Kessler, K. (2014), TEST: A Tropic, Embodied, and Situated Theory of Cognition. Topics in Cognitive Science, 6, 442460 .

Negri, G. A. L., Rumiati, R. I., Zadini, A., Ukmar, M., Mahon, B. Z., \& Caramazza, A. (2007). What is the role of motor simulation in action and object recognition? Evidence from apraxia. Cognitive Neuropsychology, 24, 8, 795-816.

Nijhoff, A. D., \& Willems, R. M. (2015). Simulating fiction: Individual differences in literature comprehension revealed with fMRI. PLoS One, 10, 2, e0116492.

Ortu, D., Allan, K., \& Donaldson, D. I. (2013). Is the N400 effect a neurophysiological index of associative relationships? Neuropsychologia, 51, 9, 1742-1748.

Papeo, L., Vallesi, A., Isaja, A., \& Rumiati, R. I. (2009). Effects of TMS on different stages of motor and non-motor verb processing in the primary motor cortex. PLoS ONE, 4, 2, e4508.

Pecher, D., Zeelenberg, R., \& Barsalou, L. W. (2003). Verifying different-modality properties for concepts produces switching costs. Psychological Science, 14, 2, 119-24. (2004). Sensorimotor simulations underlie conceptual representations: Modalityspecific effects of prior activation. Psychonomic Bulletin \& Review, 11, 1, 164-167.

Penfield, W., \& Boldrey, E. (1937). Somatic motor and sensory representations in the cerebral cortex of man as studied by electrical stimulation. Brain, 60, 389-443.

Peters, J. C., Reithler, J., Schuhmann, T., De Graaf, T., Uludağ, K., Goebel, R., \& Sack, A. T. (2015). On the feasibility of concurrent human TMS-EEG-fMRI measurements. Journal of Neurophysiology, 109, 4, 1214-1227.

Popp, M., Trumpp, N. M., \& Kiefer, M. (2016). Feature-Specific Event-Related Potential Effects to Action- and Sound-Related Verbs during Visual Word Recognition. Frontiers in Human Neuroscience, 10, 637. 
Pulvermüller, F. (1999). Words in the brain's language. Behavioral and Brain Sciences, $22,253-336$.

Pulvermuller, F., \& Garagnani, M. (2014). From sensorimotor learning to memory cells in prefrontal and temporal association cortex: a neurocomputational study of disembodiment. Cortex, 57, 1-21

Pulvermüller, F., Shtyrov, Y., \& Hauk, O. (2009). Understanding in an instant: Neurophysiological evidence for mechanistic language circuits in the brain. Brain and Language, 110, 2, 81-94.

Pulvermüller, F., Shtyrov, Y., \& Ilmoniemi, R. (2005). Brain signatures of meaning access in action word recognition. Journal of Cognitive Neuroscience, 17, 6, 884-892.

R Core Team (2015). R: A language and environment for statistical computing [Computer software manual]. Vienna, Austria. Retrieved from http://www.R-project.org/

Richter, T., \& Zwaan, R. A. (2010). Integration of perceptual information in word access. The Quarterly Journal of Experimental Psychology, 63, 1.

Rumelhart, D. E., McClelland, J. L., \& the PDP Research Group (1986). Parallel distributed processing: Explorations in the microstructure of cognition. Volume I: foundations \& volume II: Psychological and biological models. Cambridge, MA: MIT Press

Samur, D., Lai, T., Hagoort, P., \& Willems, R. M. (2015). Emotional context modulates embodied metaphor comprehension, Neuropsychologia, 78, 108-114.

Santos, A., Chaigneau, S. E., Simmons, W. K., \& Barsalou, L. W. (2011). Property generation reflects word association and situated simulation. Language and Cognition, 3, 83-119.

Sato, M., Mengarelli, M., Riggio, L., Gallese, V., \& Buccino, G. (2008). Task related modulation of the motor system during language processing. Brain and Language, 105, 83-90.

Saussure, F. d. (1916/1959). Course in general linguistics. New York: Philosophical Library.

Scerrati, E., Baroni, G., Borghi, A. M., Galatolo, R., Lugli, L., \& Nicoletti, R. (2015). The modality-switch effect: visually and aurally presented prime sentences activate our senses. Frontiers in Psychology, 6, 1668.

Scerrati, E., Lugli, L., Nicoletti, R., \& Borghi, A. M. (2016). The Multilevel ModalitySwitch Effect: What Happens When We See the Bees Buzzing and Hear the Diamonds Glistening. Psychonomic Bulletin and Review, doi:10.3758/s13423- 
016-1150-2.

Schomers, M. R., \& Pulvermüller, F. (2016). Is the sensorimotor cortex relevant for speech perception and understanding? An integrative review. Frontiers in Human Neuroscience, 10, 435.

Searle, J. (1980). Minds, Brains and Programs. Behavioral and Brain Sciences, 3, 3, $417-$ 457.

Sereno, S. C., Brewer, C. C., \& O’Donnell, P. J. (2003). Context effects in word recognition: Evidence for early interactive processing. Psychological Science, 14, 328-333.

Shetter, W. Z. (2013). Introduction to Dutch: A Practical Grammar. Dordrecht, Netherlands: Springer Science and Business Media.

Shtyrov, Y., Hauk, O., \& Pulvermüller, F. (2004). Distributed neuronal networks for encoding category-specific semantic information: the mismatch negativity to action words. European Journal of Neuroscience, 1, 4, 1083-1092.

Shtyrov, Y., Kimppa, L., Pulvermüller, F., \& Kujala, T. (2011). Event-related potentials reflecting the frequency of unattended spoken words: A neuronal index of connection strength in lexical memory circuits? NeuroImage, 55, 2, 658-668.

Simmons, W. K., Ramjee, V., Beauchamp, M. S., McRae, K., Martin, A., \& Barsalou, L. W. (2007). A common neural substrate for perceiving and knowing about color. Neuropsychologia, 45, 12, 2802-2810.

Simmons, W. K., Hamann, S. B., Harenski, C. L., Hu, X. P., \& Barsalou, L. W. (2008). fMRI evidence for word association and situated simulation in conceptual processing. Journal of Physiology, 102, 1, 106.

Skipper, J. I., Devlin, J. T., \& Lametti, D. R. (2017). The hearing ear is always found close to the speaking tongue: Review of the role of the motor system in speech perception. Brain and Language, 164, 77-105.

Solomon, K. O., \& Barsalou, L. W. (2004). Perceptual simulation in property verification. Memory \& Cognition, 32, 244-259.

Spunt, R. P., Kemmerer, D., \& Adolphs, R. (2016). The neural basis of conceptualizing the same action at different levels of abstraction. Social Cognitive \& Affective Neuroscience, 11, 7, 1141-1151.

Tettamanti, M., Buccino, G., Saccuman, M. C., Gallese, V, Danna, M., Scifo, P., ... \& Perani, D. (2005). Listening to action-related sentences activates fronto-parietal motor circuits. Journal of Cognitive Neuroscience, 17, 2, 273-281. 
Tomasello, R., Garagnani, M., Wennekers, T., \& Pulvermüller, F. (in press). Brain connections of words, perceptions and actions: A neurobiological model of spatiotemporal semantic activation in the human cortex. Neuropsychologia.

Van Dam, W. O., Brazil, I. A., Bekkering, H., \& Rueschemeyer, S.-A. (2014). Flexibility in embodied language processing: context effects in lexical access. Topics in Cognitive Science, 6, 407-424.

Van Dantzig, S., Pecher, D., Zeelenberg, R., \& Barsalou, L. W. (2008). Perceptual processing affects conceptual processing. Cognitive Science, 32, 579-590.

Van den Hoven, E., Hartung, F., Burke, M., \& Willems, R. M. (2016). Individual differences in sensitivity to style during literary reading: insights from eyetracking. Collabra, 2, 1, 25.

Van Vliet, M., Manyakov, N., Storms, G., Fias, W., Wiersema, J., \& Van Hulle, M. (2014). Response-Related Potentials during semantic priming: the effect of a speeded button response task on ERPs. PLoS One, 9, 2, e87650.

Vannuscorps, G., \& Caramazza, A. (2016). Typical action perception and interpretation without motor simulation. Proceedings of the National Academy of Sciences, 113, 86-91.

Vermeulen, N., Niedenthal, P. M., \& Luminet, O. (2007). Switching between sensory and affective systems incurs processing costs. Cognitive Science, 31, 1, 183-192.

Vigliocco, G., Vinson, D. P., Damian, M. F., \& Levelt, W. (2002). Semantic distance effects on object and action naming. Cognition 85, B61-B69.

Vukovic, V., Feurra, M., Shpektor, A., Myachykov, A., \& Shtyrov, Y. (2017). Primary motor cortex functionally contributes to language comprehension: An online rTMS study. Neuropsychologia, 96, 222-229.

Vukovic, N., \& Shtyrov, Y. (2014). Cortical motor systems are involved in secondlanguage comprehension: evidence from rapid mu-rhythm desynchronisation. Neuroimage, 102, 2, 695-703.

Whitney, C., Kirk, M., O'Sullivan, J., Lambon Ralph, M. A., \& Jefferies, E. (2012). Executive semantic processing is underpinned by a largescale neural network: revealing the contribution of left prefrontal, posterior temporal, and parietal cortex to controlled retrieval and selection using TMS. Journal of Cognitive Neuroscience, 24, 133-147.

Willems, R. M., \& Francken, J. C. (2012). Embodied cognition: Taking the next step. Frontiers in Psychology, 3, 582. 
Willems, R. M., Frank, S. L., Nijhoff, A. D., Hagoort, P., \& Van den Bosch, A. (2016). Prediction during natural language comprehension. Cerebral Cortex, 26, $6,2506-2516$.

Willems, R. M., Labruna, L., D'Esposito, M., Ivry, R., \& Casasanto, D. (2011). A functional role for the motor system in language understanding: Evidence from Theta-Burst Transcranial Magnetic Stimulation. Psychological Science, 22, 849 854.

Willems, R. M., Toni, I., Hagoort, P., \& Casasanto, D. (2010). Neural Dissociations between Action Verb Understanding and Motor Imagery. Journal of Cognitive Neuroscience, 22, 2387-2400.

Wilson, F., Papafragou, A., Bunger, A., \& Trueswell, J. T. (2011). Rapid extraction of event participants in caused motion events. In L. Carlson, C. Hölscher, \& T. Shipley (Eds.), Proceedings of the $33^{\text {rd }}$ Annual Conference of the Cognitive Science Society (pp. 1206-1211). Hillsdale, NJ: Erlbaum.

Xu, X. (2008). Individual Differences in Language Processing: An Embodied Approach. In B. C. Love, K. McRae, \& V. M. Sloutsky (Eds.), Proceedings of the 30th Annual Conference of the Cognitive Science Society (pp. 64-70). Austin, TX: Cognitive Science Society. 
Appendix 1. Linguistic variables of the target words across transition conditions. Figures in bold were controlled in the design. "Orthograp. neigh. = Orthographic neighbourhood. ${ }^{*}$ pp $=$ percentage points.

\begin{tabular}{|c|c|c|c|c|c|c|c|c|c|c|c|c|c|c|}
\hline \multicolumn{3}{|c|}{ Modality transition } & \multicolumn{4}{|c|}{ Number of letters } & \multicolumn{4}{|c|}{ Contextual diversity } & \multicolumn{4}{|c|}{ Orthograp. neigh. size } \\
\hline & & & \multicolumn{2}{|c|}{ Property } & \multicolumn{2}{|c|}{ Concept } & \multicolumn{2}{|c|}{ Property } & \multicolumn{2}{|c|}{ Concept } & \multicolumn{2}{|c|}{ Property } & \multicolumn{2}{|c|}{ Concept } \\
\hline & & & $M$ & $S D$ & $M$ & $S D$ & $M$ & $S D$ & $M$ & $S D$ & $M$ & $S D$ & $M$ & $S D$ \\
\hline \multirow{3}{*}{\multicolumn{3}{|c|}{$\begin{array}{l}\text { Visual to visual } \\
\text { Haptic to visual } \\
\text { Auditory to visual }\end{array}$}} & 7.08 & 1.83 & 6.19 & 2.24 & 1.92 & 0.72 & 2.40 & 0.85 & 3.25 & 3.76 & 4.39 & 5.09 \\
\hline & & & 7.08 & 2.14 & 7.64 & 2.72 & 1.92 & 0.66 & 2.53 & 0.70 & 3.11 & 3.28 & 2.97 & 4.75 \\
\hline & & & 7.08 & 2.27 & 6.22 & 2.15 & 1.92 & 0.71 & 2.56 & 0.77 & 4.06 & 5.69 & 3.00 & 3.41 \\
\hline & \multicolumn{4}{|c|}{ Concreteness } & \multicolumn{4}{|c|}{ Modality exclusivity* } & \multirow{2}{*}{\multicolumn{2}{|c|}{$\begin{array}{l}\text { Perceptual } \\
\text { strength }\end{array}$}} & \multirow{2}{*}{\multicolumn{2}{|c|}{$\begin{array}{l}\text { LSA } \\
\text { distance } \\
\text { property- } \\
\text { concept }\end{array}$}} & \multirow{2}{*}{\multicolumn{2}{|c|}{$\begin{array}{c}\text { True, Fals } \\
\text { trials }\end{array}$}} \\
\hline \multicolumn{3}{|c|}{ Property } & \multicolumn{2}{|c|}{ Concept } & \multicolumn{2}{|c|}{ Property } & \multicolumn{2}{|c|}{ Concept } & & & & & & \\
\hline & $M$ & $S D$ & $M$ & $S D$ & $M$ & $S D$ & $M$ & $S D$ & $M$ & $S D$ & $M$ & $S D$ & $\mathrm{~T}$ & $\mathrm{~F}$ \\
\hline $\mathrm{V} / \mathrm{V}$ & 3.35 & 0.78 & 3.12 & 0.97 & $43 \%$ & $21 \mathrm{pp}$ & $30 \%$ & $14 \mathrm{pp}$ & 3.38 & 0.39 & 0.92 & 0.10 & 17 & 19 \\
\hline $\mathrm{H} / \mathrm{V}$ & 3.11 & 0.76 & 2.73 & 0.86 & $38 \%$ & $14 \mathrm{pp}$ & $32 \%$ & $16 \mathrm{pp}$ & 3.19 & 0.41 & 0.93 & 0.06 & 22 & 14 \\
\hline $\mathrm{A} / \mathrm{V}$ & 3.17 & 0.70 & 2.91 & 0.90 & $42 \%$ & $20 \mathrm{pp}$ & $27 \%$ & $14 \mathrm{pp}$ & 3.26 & 0.52 & 0.91 & 0.11 & 17 & 19 \\
\hline
\end{tabular}

Appendix 2. Accuracy percentage per group and condition.

\begin{tabular}{c|c|c|c} 
Group & Condition & $M$ & $S D$ \\
\hline Quick & Visual to visual & $61 \%$ & $49 \mathrm{pp}$ \\
Quick & Haptic to visual & $56 \%$ & $50 \mathrm{pp}$ \\
Quick & Auditory to visual & $66 \%$ & $47 \mathrm{pp}$ \\
Self-paced & Visual to visual & $63 \%$ & $48 \mathrm{pp}$ \\
Self-paced & Haptic to visual & $57 \%$ & $49 \mathrm{pp}$ \\
Self-paced & Auditory to visual & $67 \%$ & $47 \mathrm{pp}$ \\
Null & Visual to visual & $64 \%$ & $48 \mathrm{pp}$ \\
Null & Haptic to visual & $57 \%$ & $50 \mathrm{pp}$ \\
Null & Auditory to visual & $73 \%$ & $45 \mathrm{pp}$ \\
\hline
\end{tabular}

